Flat deformation of a spacetime admitting two commuting Killing fields

This article has been downloaded from IOPscience. Please scroll down to see the full text article.

2010 Class. Quantum Grav. 27245006

(http://iopscience.iop.org/0264-9381/27/24/245006)

View the table of contents for this issue, or go to the journal homepage for more

Download details:

IP Address: 161.116.80.144

The article was downloaded on 15/03/2013 at 09:40

Please note that terms and conditions apply. 


\title{
Flat deformation of a spacetime admitting two commuting Killing fields
}

\author{
Josep Llosa $^{1}$ and Jaume Carot ${ }^{2}$ \\ ${ }^{1}$ Departament de Física Fonamental, Universitat de Barcelona, Barcelona, Spain \\ 2 Departament de Física, Universitat de les Illes Balears, Palma de Mallorca, Illes Balears, Spain \\ E-mail: pitu.llosa@ub.edu
}

Received 14 May 2010, in final form 5 October 2010

Published 22 November 2010

Online at stacks.iop.org/CQG/27/245006

\begin{abstract}
It is shown that, given an analytic Lorentzian metric on a 4-manifold, $g_{a b}$, which admits two Killing vector fields, there exists a local deformation law $\eta_{a b}=a g_{a b}+b H_{a b}$, where $H_{a b}$ is a two-dimensional projector, such that $\eta_{a b}$ is flat and admits the same Killing vectors. We also characterize the particular case when the projector $H_{a b}$ coincides with the quotient metric. We apply some of our results to general stationary axisymmetric spacetimes.
\end{abstract}

PACS numbers: $04.20 . \mathrm{Cv}, 02.40 . \mathrm{Hw}, 02.40 . \mathrm{Ky}$

\section{Introduction}

On the basis that the number of degrees of freedom of a semi-Riemannian metric on an $n$-manifold is $n(n-1) / 2$, in [1] we proved that

Theorem 1 (deformation theorem). Let $\mathcal{V}_{n}$ be an analytic manifold with a semi-Riemannian analytic metric, $g$. Locally there always exists a 2-form $F$ and a scalar function $\beta$ such that

(a) they meet a previously chosen arbitrary scalar constraint $\Psi(\beta, F)=0$

(b) and the (semi)Riemannian deformed metric

$$
\bar{g}_{a b}=\beta g_{a b}-\epsilon F_{a b}^{2}, \quad \text { with } \quad F_{a b}^{2}:=g^{c d} F_{a c} F_{d b}, \quad|\epsilon|=1,
$$

has a constant curvature whose value can be arbitrarily prescribed.

This can be done in an infinite number of ways, that is, $\beta$ and $F$ are not uniquely determined by $g$ and $\psi$.

A corollary is that, locally, any analytic spacetime metric can be obtained by deforming a flat metric $\eta$ according to

$$
g_{a b}=\lambda(F)+\epsilon \eta^{c d} F_{a c} F_{d b}, \quad F \in \Lambda^{2} \mathcal{M}_{4},
$$

where $\lambda$ is a scalar function of $F$. 
In [2] we realized that for $n=4$ theorem 1 could be stated in an equivalent way where the arbitrary constraint $\Psi$ was not necessary. It reads

Theorem 2. Let $g_{a b}$ be a Lorentzian analytic metric on a spacetime $\mathcal{M}$. Locally there exist two scalars, $a$ and $b$, and a two-dimensional projector $H_{a b}$, i.e. $H_{a b} g^{b c} H_{c d}=H_{a d}$ and $\left(H_{a b} g^{a b}\right)=2$, such that the deformed metric

$$
\eta_{a b}:=a g_{a b}+b H_{a b}
$$

is flat.

We call this formula the deformation law associated with $\left(a, b, H_{a b}\right)$.

This has an immediate application to general relativity because from equation (1) we have that

$$
g_{a b}=\varphi \eta_{a b}+\rho H_{a b}, \quad \text { with } \quad \varphi=\frac{1}{a}, \quad \rho=-\frac{b}{a},
$$

and this suggests the possibility of representing the gravitational field by the two scalars $\varphi$ and $\rho$ and the four independent components of the 2-projector $H_{a b}$ instead of the ten standard gravitational potentials $g_{a b}$. In this way we could avoid the huge gauge freedom associated with the group of diffeomorphisms which permits to assign some prescribed values to four among the ten quantities $g_{a b}{ }^{3}$

Note that using $\left(\varphi, \rho, H_{a b}\right)$ as 'gravitational potentials' implies the choice of a preferred flat metric, namely $\eta_{a b}$. This breaks the diffeomorphism invariance of the Lagrangian (the transcription of Einstein-Hilbert's in terms of these potentials) and the only remaining invariance corresponds to the Lie group of motions of the flat metric $\eta_{a b}$. By applying Noether's theorem, this would yield a canonical definition of the energy-momentum tensor of the gravitational field. These potentials could also be considered as a basis for a canonical quantization of the gravitational field.

The two-dimensional projector $H_{a b}$ defines an almost-product structure $[4,5] g_{a b}=$ $H_{a b}+K_{a b}$; then the above result implies that given $g_{a b}$ an almost-product structure exists such that differently scaling the plane $H_{a b}$, by a factor $\varphi=a+b$, and the plane $K_{a b}$, by a factor $a$, the resulting metric $\eta_{a b}:=(a+b) H_{a b}+a K_{a b}$ is flat. In this sense it can be said that the deformation law (1) is a biconformal transformation [3].

Let $\left\{\omega_{a}^{1}, \omega_{a}^{2}, \omega_{a}^{3}, \omega_{a}^{4}\right\}$ be a $g$-orthonormal frame adapted to the above almost-product structure, that is, $H_{a b}=\omega_{a}^{1} \otimes \omega_{b}^{1}+\omega_{a}^{2} \otimes \omega_{b}^{2}$ and $K_{a b}=\omega_{a}^{3} \otimes \omega_{b}^{3}+\omega_{a}^{4} \otimes \omega_{b}^{4}$, and let $U_{a b}:=\omega_{a}^{1} \wedge \omega_{b}^{2} \in \Lambda^{2} \mathcal{M}$. As $U_{a b}^{2}:=U_{a}^{c} U_{c b}=H_{a b}$, it follows immediately that in four dimensions the deformation theorem can be stated as

Theorem 3. Let $\mathcal{V}_{4}$ be an analytic spacetime with metric $g$. Locally there always exists a decomposable 2-form $U$ and two scalar functions $\beta$ and $\rho$ such that the deformed metric

$$
\bar{g}_{a b}=\beta g_{a b}+\rho U_{a b}^{2}
$$

is flat or has constant curvature.

The gravitational field is thus written in terms of two scalars and a decomposable 2-form (a 2-plane or a blade). This recalls the Rainich's formalism for the electromagnetic field [6], where the field is characterized by two invariants and a 2-plane. Then, Maxwell equations yield a system of decoupled equations for the invariants and the 2-plane structure. One could wonder whether a similar treatment is possible for the gravitational field.

3 It seems however that some gauge is left because the deformation parameters $\left(a, b, H_{a b}\right)$ are by no means uniquely determined by $g_{a b}$. 
As we have said, given $g_{a b}$, the deformation law transforming it into a flat metric is not unique. In the case that $g_{a b}$ has some symmetries, they could help to reduce this ambiguity, namely by requiring that the deformation law (1) is compatible with those symmetries. In [2] we proved that this is feasible, that is, if $g_{a b}$ admits a Killing field $X^{a}$, then a deformation law can be found such that $\eta_{a b}$ also admits $X^{a}$ as a Killing field.

Assume now that $g_{a b}$ admits a wider Lie algebra $\mathcal{G}$ of Killing fields, i.e. $\operatorname{dim} \mathcal{G}>1$. Is it possible to find a deformation law such that $\eta_{a b}$ admits any $X \in \mathcal{G}$ as a Killing field? Note that, as $\eta_{a b}$ is flat, the Lie algebra of its isometries is maximal, i.e. the Poincaré algebra $\mathcal{P}$. Therefore, in order that the answer to the question above is affirmative, it is necessary that $\mathcal{G} \subset \mathcal{P}$. We thus advance the following conjecture:

if $g_{a b}$ admits a Killing algebra $\mathcal{G}$ and $\mathcal{G} \subset \mathcal{P}$, then there exist deformation laws such as (1) such that $\eta_{a b}$ is flat and any $X \in \mathcal{G}$ is a Killing field of $\eta_{a b}$.

The problem we shall tackle in this paper is little bit simpler: we shall consider an analytic ${ }^{4}$ metric $g_{a b}$ admitting two commuting Killing fields and we shall see that a deformation law (1) exists such that $\eta_{a b}$ is flat and admits the same Killing fields. We shall confine ourselves to the case in which the metric induced on the plane spanned by the Killing vectors is non-degenerate and hyperbolic, that is, timelike orbits (whereas the elliptic case can be dealt in a similar way, the degenerate case is quite different).

At this point, the relevance of the almost-product structure defined by the projector $H_{b}^{a}$ (and the very fact of this being a projector) should be emphasized. This feature is largely responsible for the complexity of the proof of the main theorem in this paper (see theorem 4), but at the same time it confers the result, a deep geometrical interest.

The paper is structured as follows: in section 2 we present the formalism and prove some intermediate results concerning spacetimes admitting two commuting Killing fields ${ }^{5}$; we also assume that the quotient space is a 2-manifold. These results are then applied in section 3 to prove the flat deformation theorem. The formalism allows a reformulation of the proof in the quotient 2-manifold, so that a dimensional reduction occurs. At this point it is worth stressing the usefulness of the so-called reconstruction problem (see section 2.3), which consists in reconstructing a flat metric in the spacetime from a given quotient metric. In section 4 , we study the especially simple case when the almost-product structure implicit in the deformation law coincides with the almost-product structure induced by the Killing fields and we apply the above results to the case of stationary axisymmetric spacetimes.

\section{Spacetimes admitting two commuting Killing vectors}

Let $\mathcal{M}$ be a spacetime with a metric $\eta_{a b}$ admitting two commuting Killing vectors $X_{A}^{a}$,

$$
\mathcal{L}_{X_{A}} \eta_{a b}=0, \quad A=1,2 .
$$

Note that, at this point, $\eta_{a b}$ does not designate necessarily a flat metric. Through any point $x \in \mathcal{M}$, there is an integral submanifold $\mathcal{V}_{x}$, i.e. $T_{x} \mathcal{V}_{x}=\operatorname{span}\left\{X_{A}^{a}, A=1,2\right\}$, which we call the orbit trough $x$. Let $\left\{e_{a}, a=1, \ldots, 4\right\}$ be a base in $T_{x} \mathcal{M}$ and $\left\{\omega^{b}, b=1, \ldots, 4\right\}$ its dual base. We denote by $\lambda_{A B}$ the metric products:

$\lambda_{A B}:=X_{A}^{a} \xi_{B a} \quad$ and $\quad \lambda^{A B} \lambda_{B D}=\delta_{D}^{A}, \quad$ where $\quad \xi_{A a}:=\eta_{a b} X_{A}^{b}$,

and define

4 As the Cauchy-Kovalewski theorem is invoked at some point in the proof, the validity of the results presented here is restricted to the analytic category.

5 With a different notation, this formalism was developed in [8] and we present it here in a way suited to our purposes. 


$$
\xi^{A a}:=\lambda^{A B} X_{B}^{a} \quad \text { and } \quad X_{B}^{a} \xi_{a}^{A}=\delta_{B}^{A},
$$

that is, $\xi_{a}^{A}, A=1,2$, is the dual base for $X_{A}^{a}, A=1,2$, on $T_{x} \mathcal{V}_{x}$.

The metric induced by $\eta_{a b}$ on the orbit $\mathcal{V}_{x}$ is $\lambda_{A B} \xi_{a}^{A} \xi_{b}^{B}=\xi_{A a} \xi_{b}^{A}$ which, as already mentioned, will be assumed hyperbolic, that is,

$$
\operatorname{det}\left(\lambda_{A B}\right)=:-\frac{\tau^{2}}{2}<0
$$

and that, in a terminology borrowed from principal bundle theory [10], we shall call the vertical metric. Note that, from its definition and the fact that the Killing vectors commute, it is immediate to see that $\lambda_{A B}$ is preserved by $X_{A}$, i.e. $\mathcal{L}_{X_{A}} \lambda_{A B}=0$.

Let us assume that the set of all orbits of $X_{A}^{a}, A=1,2$, is a 2-manifold, i.e. the quotient manifold $\mathcal{S}$. The tensor

$$
h_{b}^{a}:=\delta_{b}^{a}-X_{A}^{a} \xi_{b}^{A}
$$

projects then vectors in $T \mathcal{M}$ onto vectors that are orthogonal to the orbits. Again, in analogy with the principal bundle terminology, vectors that are orthogonal to the orbits will be called horizontal.

There is a one-to-one correspondence [7] between tensor fields $T_{b \ldots}^{\prime a \ldots}$ on $\mathcal{S}$ and horizontal tensor fields on $\mathcal{M}$, i.e. those $T_{b \ldots}^{a \ldots}$ fulfilling

$X_{B}^{b} T_{b \ldots}^{a \ldots}=0, \quad \xi_{a}^{A} T_{b \ldots}^{a \ldots}=0 \quad$ and $\quad \mathcal{L}_{X_{A}} T_{b \ldots}^{a \ldots}=0, \quad A, B=1,2$, that is, tensor fields that are horizontal and Lie-constant along $X_{A}^{a}$. Following Geroch [7] while it is useful conceptually to have the two-dimensional manifold $\mathcal{S}$, it plays no further logical role in the formalism. We shall hereafter drop the primes: we shall continue to speak of tensor fields being on $\mathcal{S}$, merely as a shorthand way of saying that the field (formally, on $\mathcal{M})$ satisfies (6).

From $\mathcal{L}_{X_{A}} \eta_{a b}=0$ and the fact that the Killing vectors commute, it follows that the horizontal metric

$$
h_{a b}:=\eta_{a b}-\xi_{A a} \xi_{b}^{A}, \quad \text { i. e. } \quad h_{a b}=\eta_{a c} h_{b}^{c},
$$

is preserved by $X_{A}, A=1,2$. By the above-mentioned correspondence, it induces a metric on $\mathcal{S}$ and, as the vertical metric is hyperbolic, $h_{a b}$ is elliptic. We shall denote the inverse horizontal metric as $h^{a b}$; one then has $h^{a b}:=\eta^{a b}-\xi^{A a} X_{A}^{b}$ and $h^{a b} h_{b c}=h_{c}^{a}$.

\subsection{The Killing equation}

From $\mathcal{L}_{X_{A}} \eta_{a b}=0$ it follows that $\nabla_{a} \xi_{A b}$ is skewsymmetric, or

$$
\nabla_{a} \xi_{A b}=\frac{1}{2}\left(\mathrm{~d} \xi_{A}\right)_{a b} .
$$

As the Killing vectors commute, it follows easily that

$$
X_{A}^{a} \lambda_{B C \mid a}=0 \quad \text { and } \quad X_{A}^{a} \tau_{a}=0,
$$

where $\mathrm{d} \tau=\tau_{a} \omega^{a}$ and a stroke $\mid$ denotes differentiation. Hence, $\lambda_{B C}$ and $\tau$ are functions on $\mathcal{S}$ and $\lambda_{B C \mid a}, \tau_{b}$ are 1 -forms on $\mathcal{S}$.

A further consequence of (9) and the commutativity of the Killing vectors is that $\mathcal{L}_{X_{B}} \xi_{a}^{A}=0$, whence it follows that

$\mathrm{i}\left(X_{B}\right) \mathrm{d} \xi^{A}=0 \quad$ and $\quad \mathcal{L}_{X_{B}} \mathrm{~d} \xi_{a}^{A}=0 ; \quad$ therefore, $\quad \mathrm{d} \xi^{A} \in \Lambda^{2} \mathcal{S}$.

As $\mathcal{S}$ has only two dimensions, $\mathrm{d} \xi^{A}=\theta^{A} \epsilon$, where $\epsilon$ is the volume tensor (see appendix A) and we call the scalar $\theta^{A}$, the $t w i s t$ of $X_{A}$ with respect to $\eta_{a b}$ or $\eta$-twist. Then, including (8), we have that

$$
\begin{aligned}
& \nabla_{c} \xi_{A d}=\lambda_{A B \mid[c} \xi_{d]}^{B}+\frac{1}{2} \theta_{A} \epsilon_{c d} \quad \text { and } \quad \nabla_{c} \xi_{d}^{A}=-\lambda^{A D} \lambda_{D B \mid(d} \xi_{c)}^{B}+\frac{1}{2} \theta^{A} \epsilon_{c d} \\
& \text { with } \theta_{A}:=\lambda_{A B} \theta^{B} .
\end{aligned}
$$




\subsection{The Levi-Civita connection on the quotient manifold $\mathcal{S}$ and the Riemann tensor}

Given a horizontal tensor $T_{b \ldots}^{a \ldots}$ we define

$$
D_{c} T_{b \ldots}^{a \ldots}:=h_{c}^{d} h_{e}^{a} h_{b}^{f} \nabla_{d} T_{f \ldots}^{e \ldots} .
$$

From (A.5), it follows that for a horizontal vector $w_{b}$,

$$
D_{a} w^{b}=\nabla_{a} w^{b}+w^{d}\left[-\frac{1}{2} X_{A}^{A} \lambda_{A B \mid d} \xi^{B b}+\frac{1}{2} \theta_{A}\left(\xi_{a}^{A} \epsilon_{d}^{b}+\xi^{A b} \epsilon_{a d}\right)\right] .
$$

It can be easily proved that $D$ is a symmetric linear connection on the quotient manifold. Moreover, since $D_{a} h_{b c}=0$, it is the Levi-Civita connection for $h_{b c}$ in $\mathcal{S}$.

The Riemann tensor $\mathcal{R}_{c d a b}$ for the connection $D$ can be derived from the Ricci identities, $D_{a} D_{b} w^{c}-D_{b} D_{a} w^{c}=w^{d} \mathcal{R}_{d a b}^{c}$, and one thus obtains

$$
\mathcal{R}_{d c a b}=R_{d c a b}^{\perp}-\frac{1}{2} \theta_{A} \theta^{A}\left(\epsilon_{a b} \epsilon_{d c}-\epsilon_{d[a} \epsilon_{b] c}\right),
$$

where $R_{d c a b}^{\perp}:=h_{a}^{p} h_{b}^{q} h_{c}^{n} h_{d}^{m} R_{m n p q}$.

Due to the symmetries of the Riemann tensor and the low dimensionality, we also have that

$$
\mathcal{R}_{d c a b}=\mathcal{R} h_{d[a} h_{b] c}=\frac{\mathcal{R}}{2} \epsilon_{d c} \epsilon_{a b}
$$

and a similar expression for $R_{d c a b}^{\perp}$. Hence, equation (13) implies that

$h^{d a} h^{b c} R_{d c a b}=\mathcal{R}-\frac{3}{2} \theta^{A} \theta_{A} \quad$ and $\quad R_{d c a b}^{\perp}=\left(\mathcal{R}-\frac{3}{2} \theta^{A} \theta_{A}\right) h_{d[a} h_{b] c}$.

To derive the remaining components of $R_{c d a b}$, namely those having some vertical indices, we use that, since $X_{D}^{b}$ is a Killing vector [9],

$$
R_{D c a b}:=X_{D}^{d} R_{d c a b}=\nabla_{c} \nabla_{a} \xi_{D b}
$$

and, after some algebra we obtain

$R_{D c a b}^{\perp}=\frac{1}{2}\left(D_{c} \theta_{D}+\frac{1}{2} \theta^{E} \lambda_{D E \mid c}\right) \epsilon_{a b}$,

$R_{D c A b}^{\perp}=-\frac{1}{2}\left(D_{b} \lambda_{A D \mid c}-\frac{1}{2} \lambda^{T E} \lambda_{A E \mid c} \lambda_{T D \mid b}-\frac{1}{2} \theta_{A} \theta_{D} h_{b c}\right)$,

$R_{D C A b}^{\perp}=-\frac{1}{2} \theta_{[C} \lambda_{D] A \mid d} \epsilon_{b}^{d} \quad$ and $\quad R_{D C A B}=-\frac{1}{2} h^{b c} \lambda_{C[B \mid c} \lambda_{A] D \mid b}$.

The Riemann tensor for $\eta_{a b}$ can be reconstructed from these components according to

$$
\begin{aligned}
R_{d c a b}=R_{1} \epsilon_{d c} & \epsilon_{a b}+R_{2} \Omega_{d c} \Omega_{a b}+2 P_{A[b} \xi_{a]}^{A} \epsilon_{d c}+2 P_{A[c} \xi_{d]}^{A} \epsilon_{a b} \\
& +2 Q_{A[b} \xi_{a]}^{A} \Omega_{d c}+2 Q_{A[c} \xi_{d]}^{A} \Omega_{a b}+4 \xi_{[d}^{D} P_{D c] A[b} \xi_{a]}^{A} \\
& +\frac{1}{2} R_{3}\left(2 \epsilon_{d c} \Omega_{a b}-\epsilon_{d a} \Omega_{b c}-\epsilon_{d b} \Omega_{c a}+2 \Omega_{d c} \epsilon_{a b}-\Omega_{d a} \epsilon_{b c}-\Omega_{d b} \epsilon_{c a}\right)
\end{aligned}
$$

where $\Omega_{a b}$ and $\epsilon_{d c}$ are respectively defined in (A.1) and (A.2) (see appendix A) and

$$
\begin{aligned}
& R_{1}=\frac{1}{4} R_{d c a b} \epsilon^{d c} \epsilon^{a b}=\frac{1}{2}\left(\mathcal{R}-\frac{3}{2} \theta^{A} \theta_{A}\right) \\
& R_{2}=R_{d c a b} \Omega^{d c} \Omega^{a b}=-\frac{1}{\tau^{2}}\left(\lambda_{11 \mid c} \lambda_{22 \mid b}-\lambda_{12 \mid c} \lambda_{12 \mid b}\right) h^{b c} \\
& R_{3}=\frac{1}{2} R_{d c a b} \Omega^{d c} \epsilon^{a b}=\frac{1}{2 \tau} \epsilon^{b c} \lambda^{T E} \lambda_{1 E \mid c} \lambda_{2 T \mid b} \\
& P_{A b}=\frac{1}{2}\left(R_{d c A b} \epsilon^{d c}\right)^{\perp}=\frac{1}{2}\left(D_{b} \theta_{A}+\frac{1}{2} \theta^{T} \lambda_{T A \mid b}\right)
\end{aligned}
$$




$$
\begin{aligned}
& Q_{A b}=\left(R_{d c A b} \Omega^{d c}\right)^{\perp}=-\frac{1}{\tau} \theta_{[1} \lambda_{2] A \mid c} \epsilon_{b}^{c} \\
& P_{D c A b}=R_{D(c|A| b)}^{\perp}=-\frac{1}{2}\left(D_{b} \lambda_{A D \mid c}-\frac{1}{2} \lambda^{T E} \lambda_{A E \mid(c} \lambda_{T D \mid b)}-\frac{1}{2} \theta_{A} \theta_{D} h_{b c}\right) .
\end{aligned}
$$

It is trivial to see that in expression (19) the first Bianchi identity is componentwise satisfied. Equations (20)-(25) are relations between the quotient metric and the covariant kinematical invariants of the Killing fields, on the one hand, and the ambient metric on the other. They must also be taken as equations to solve in the so-called reconstruction problem (see the next section).

\subsection{The reconstruction problem}

It consists in reconstructing an ambient metric $\eta_{a b}$ from a given quotient metric $h_{a b}$ provided that $\eta_{a b}$ admits two Killing vectors $X_{A}^{b}, A=1,2$. It is particularly an interesting case in which the final ambient metric is required to have some prescribed geometric properties, e.g. being flat, which is the case we shall ultimately be interested in.

It is easy to prove that giving a metric $\eta_{a b}$ on $\mathcal{M}$ is equivalent to providing:

(a.i) two covectors $\xi_{A a} \in \Lambda^{1} \mathcal{M}$ such that $\mathcal{L}_{X_{B}} \xi_{A a}=0$ and that $\lambda_{A B}:=\xi_{A a} X_{B}^{a}$ is a nondegenerate matrix, and

(a.ii) the quotient metric on $\mathcal{S}$. (The signatures of both $h_{a b}$ and $\lambda_{A B}$ must be chosen so that the signature of $\eta_{a b}$ is $(+3,-1)$.)

On their turn these conditions are equivalent to giving

(b.i) two covectors $\xi_{a}^{A} \in \Lambda^{1} \mathcal{M}$ such that $\mathcal{L}_{X_{B}} \xi_{A a}=0$ and that $\xi_{a}^{A} X_{B}^{a}=\delta_{A}^{B}$,

(b.ii) a 2-squared symmetric non-degenerate matrix $\lambda_{A B} \in \Lambda^{0} \mathcal{S}$ and

(b.iii) the quotient metric on $\mathcal{S}$.

\subsection{Reconstructing a flat metric with two prescribed Killing vectors}

Assume now that we want the ambient metric to be flat. Are there any further restrictions on $h_{a b}, \xi_{a}^{A}$ and $\lambda_{A B}$ that are derived from the flatness of $\eta_{a b}$ ?

As $\eta_{a b} X_{B}^{a}=\xi_{B a}$ and $X_{A}^{a}, A=1,2$, are Killing vectors, the results derived in section 2.1 apply. Therefore, $\mathcal{L}_{X_{B}} \xi_{a}^{A}=0, \mathcal{L}_{X_{B}} \lambda_{A B}=0$ and equations similar to (11) do hold. Thus, although $\lambda_{A B} \in \Lambda^{0}(\mathcal{S}), \xi_{A a}$ and $\xi_{a}^{A}$ are not covectors on $\mathcal{S}$ because they are not orthogonal to $X_{B}^{b}$. Let us assume however that we are given two covectors $\bar{\xi}_{a}^{A} \in \Lambda^{1}(\mathcal{M})$ such that $\bar{\xi}_{A b} X_{B}^{b}=\delta_{B}^{A}, A, B=1,2$, and that $\mathcal{L}_{X_{B}} \bar{\xi}_{a}^{A}=0$. Then $\xi_{a}^{A}$ can be written as

$$
\xi_{a}^{A}=\bar{\xi}_{a}^{A}+\kappa_{a}^{A}, \quad \text { with } \quad \kappa_{a}^{A} \in \Lambda^{1}(\mathcal{S}) .
$$

We shall call $\kappa_{a}^{A}$ the shift covectors, differentiating and taking (11) into account, we arrive at

$$
\theta^{A} \epsilon=\mathrm{d} \bar{\xi}^{A}+\mathrm{d} \kappa^{A} \text {. }
$$

Bearing this result in mind, expressions (20)-(25) imply a second-order partial differential system on the variables $\lambda_{A B}, \kappa_{a}^{A} \mathrm{i} h_{a b}$, namely

$$
R_{1}=R_{2}=R_{3}, \quad P_{A b}=Q_{A b}=0, \quad P_{D c A b}=0,
$$

which has to be solved on $\mathcal{S}$ and the solutions are to be used as the data (b.i) to (b.iii) necessary to reconstruct $\eta_{a b}$.

In appendix B we prove that equations (28) imply that $h_{a b}, \xi_{a}^{A}$ and $\lambda_{A B}$ are constrained by the following conditions. 
- The horizontal metric $h_{a b}$ must be either (i) flat or, if not, (ii) its Ricci scalar must satisfy

$$
D_{b c} \mathcal{R}^{-1 / 4}+\frac{1}{6} \mathcal{R}^{3 / 4} h_{b c}=0
$$

- In case (i), take $\theta_{A}=0$ and

(i.a) either take (equation (B.18), $q=0$ )

$$
\lambda_{A B}=\frac{\tau_{0}}{\sqrt{2}}\left[\hat{\lambda}_{A B}+F \hat{q}_{A B}\right] \quad \text { with } \quad D_{a b} F=0,
$$

where $\hat{\lambda}_{A B}$ and $\hat{q}_{A B}$ are constant matrices fulfilling $\hat{q}_{A B} \hat{\lambda}^{A B}=\hat{q}_{A B} \hat{\lambda}^{B C} \hat{q}_{C D}=0$, and $\operatorname{det}\left(\hat{\lambda}_{A B}\right)=-1$.

(i.b) or take (equation (B.24), $q=1$ )

$$
\lambda_{A B}=\frac{1}{\sqrt{2}}\left(-\Phi_{-}^{2} \hat{m}_{A} \hat{m}_{B}+\Phi_{+}^{2} \hat{n}_{A} \hat{n}_{B}\right),
$$

with $\hat{m}_{2} \hat{n}_{1}-\hat{m}_{1} \hat{n}_{2}=1$, where

$$
\mathcal{F}_{b c}^{ \pm}:=D_{b c} \Phi_{ \pm}=0 \quad \text { with } \quad R_{2}:=h^{a b} D_{a} \Phi_{+} D_{b} \Phi_{-}=0 .
$$

- In case (ii) choose two constants $\alpha \neq 0$ and $C \neq 0$ and take

$$
\tau=\left(-\frac{3 \alpha C^{2}}{\mathcal{R}}\right)^{1 / 4}, \quad \theta=\frac{C}{\tau}=\left(-\frac{C^{2} \mathcal{R}}{3 \alpha}\right)^{1 / 4} ;
$$

then choose $k_{A}$ and $\hat{\lambda}^{A B}$ such that $\hat{\lambda}^{A B} k_{A} k_{B}=\alpha$ and take

$$
\begin{aligned}
& \theta_{A}=k_{A} \theta, \quad \theta^{A}=-\frac{2 C}{\tau^{3}} \hat{\lambda}^{A B} k_{B}, \\
& \lambda_{A B}=k_{A} k_{B} \lambda+\hat{\lambda}_{A B}, \quad \lambda:=-\frac{1}{\alpha}\left(\frac{\tau^{2}}{2}+\delta_{0}\right) .
\end{aligned}
$$

In both cases we still have to determine $\xi^{A} \in \Lambda^{1} \mathcal{M}$. To this aim, we first choose two 1-forms $\bar{\xi}^{A} \in \Lambda^{1} \mathcal{M}$ such that $\mathcal{L}_{X_{B}} \bar{\xi}^{A}=0$ and $X_{B}^{a} \bar{\xi}_{a}^{A}=\delta_{B}^{A}$; it is obvious that $\mathrm{d} \bar{\xi}^{A} \in \Lambda^{2} \mathcal{S}$. The shift covector, $\kappa^{A}:=\xi^{A}-\bar{\xi}^{A} \in \Lambda^{1} \mathcal{S}$, can be determined by solving

$$
\mathrm{d} \kappa^{A}=\theta^{A} \epsilon-\mathrm{d} \bar{\xi}^{A}
$$

which follows from (11) and is always integrable due to the fact that $\operatorname{dim} \mathcal{S}=2$.

\section{Flat deformation}

The central result of this paper is the following theorem.

Theorem 4. Let $g_{a b}$ be a Lorentzian metric admitting two commuting Killing vector fields, $X_{A}^{a}, A=1,2$. Then, there exist two functions $a, b$ and an elliptic two-dimensional projector $H_{b}^{a}$ such that the deformed metric

$$
\eta_{a b}:=a g_{a b}+b H_{a b}, \quad \text { where } \quad H_{a b}:=g_{a c} H_{b}^{c},
$$

is flat and admits $X_{A}^{a}, A=1,2$, as Killing vector fields with vanishing $\eta$-twists.

The proof spreads all over the present section and the following lemma allows for a dimensional reduction of the problem. (See [2] for a proof.)

Lemma 1. Let $X^{a}$ be a Killing vector for $g_{a b}$ and let $\eta_{a b}$ be defined by (34) with $b \neq 0$; then

$$
\mathcal{L}_{X} \eta_{a b}=0 \quad \Leftrightarrow \quad \mathcal{L}_{X} a=\mathcal{L}_{X} b=0 \quad \text { and } \quad \mathcal{L}_{X} H_{a b}=0 .
$$

Therefore, $a, b \in \Lambda^{0} \mathcal{S}$. 
Then, it is useful to consider that, with respect to the Killing vectors, both metrics $g_{a b}$ and $\eta_{a b}$ split into their horizontal and vertical parts as follows:

$$
g_{a b}=\bar{h}_{a b}+\bar{\lambda}_{A B} \bar{\xi}_{a}^{A} \bar{\xi}_{b}^{B}, \quad \eta_{a b}=h_{a b}+\lambda_{A B} \xi_{a}^{A} \xi_{b}^{B}
$$

where $\bar{h}_{a b}$ and $h_{a b}$ are the respective quotient metrics and $\bar{\lambda}_{A B} \bar{\xi}_{a}^{A} \bar{\xi}_{b}^{B}$ and $\lambda_{A B} \xi_{a}^{A} \xi_{b}^{B}$ are the respective metrics on the orbits. $\xi_{a}^{A}$ and $\lambda_{A B}$ are defined as in (3) and similar relations hold for $\bar{\xi}_{a}^{A}$ and $\bar{\lambda}_{A B}$, that is,

$\bar{\xi}_{A a}:=g_{a b} X_{A}^{b}, \quad \bar{\lambda}_{A B}:=g_{a b} X_{A}^{a} X_{B}^{b}, \quad \bar{\xi}_{a}^{A}:=\bar{\lambda}^{A B} \bar{\xi}_{B a} \quad$ and $\quad \bar{\lambda}^{A B} \bar{\lambda}_{B C}=\delta_{C}^{A}$.

Since $X_{A}^{a}$ are commuting Killing vectors for both $g_{a b}$ and $\eta_{a b}$, we have that $\mathcal{L}_{X_{A}} \xi_{a}^{B}=$ $\mathcal{L}_{X_{A}} \bar{\xi}_{a}^{B}=0$ and, as a consequence, we can introduce the shift covectors

$$
\kappa_{a}^{A}:=\xi_{a}^{A}-\xi_{a}^{A} \in \Lambda^{1} \mathcal{S} .
$$

The inverse (contravariant) metrics are, respectively,

$$
g^{a b}=\bar{h}^{a b}+\bar{\lambda}^{A B} X_{A}^{a} X_{B}^{b} \quad \text { and } \quad \eta^{a b}=h^{a b}+\lambda^{A B} X_{A}^{a} X_{B}^{b} .
$$

As commented above-conditions (b.i) to (b.iii) in subsection 2.3-determining $\eta_{a b}$ is equivalent to finding an elliptic horizontal metric $h_{a b}$, the hyperbolic matrix $\lambda_{A B}, A, B=1,2$, and two covectors $\xi_{a}^{A}$ such that $\xi_{a}^{A} X_{B}^{a}=\delta_{B}^{A}$.

Furthermore, we want both metrics $\eta_{a b}$ and $g_{a b}$ to be related by the deformation law (34), i.e. $\frac{1}{b} g^{a c}\left(\eta_{c b}-a g_{a b}\right)$ has to be a projector. This can be easily proved to be equivalent to

$$
\eta_{a b}-(2 a+b) g_{a b}+a(a+b) g_{a c} g_{b d} \eta^{c d}=0
$$

which, using (37), can be separated in its horizontal, vertical and cross parts, respectively,

$$
\begin{aligned}
& h_{a b}+\lambda_{A B} \kappa_{a}^{A} \kappa_{b}^{B}-(2 a+b) \bar{h}_{a b}+a(a+b) \bar{h}_{a c} \bar{h}_{b d} h^{c d}=0 \\
& \lambda_{A B}-(2 a+b) \bar{\lambda}_{A B}+a(a+b) \bar{\lambda}_{A C} \bar{\lambda}_{B D}\left(\lambda^{D C}+h^{c d} \kappa_{d}^{D} \kappa_{c}^{C}\right)=0 \\
& \lambda_{A B} \kappa_{b}^{B}-a(a+b) \lambda_{A B} \kappa_{d}^{B} h^{c d} \bar{h}_{b c}=0 .
\end{aligned}
$$

According to what has been said about the reconstruction problem, if the deformed metric $\eta_{a b}$ has to be flat, $h_{a b}, \kappa_{a}^{A}$ and $\lambda_{A B}$ must fulfil the conditions detailed there. Particularly, as we want the $\eta$-twists to vanish, the shift covectors must fulfil

$$
\mathrm{d} \kappa^{A}=-\bar{\theta}^{A} \bar{\epsilon}
$$

where $\bar{\theta}^{A}$ are the $g$-twists, $\bar{\epsilon}$ is the $\bar{h}$-volume tensor and (37) has been included.

We can besides decide to be in the case (i.b) - see section 2.4-and take $\bar{h}_{a b}$ flat and $\lambda_{A B}$ given by (31). The equations to solve are then (32), (42) and $\mathcal{R}=0$.

In appendix C-equations (C.1), (C.2), (C.14) and (C.17) - we prove that, on the basis of relations (39)-(41), $h_{a b}, \lambda_{A B}$ and $\kappa_{b}^{A}$ can be written in terms of five unknown functions $\Phi_{ \pm}, a$, $b$ and $\psi$. Then, substituting those expressions into (32), (42) and $\mathcal{R}=0$, the following partial differential system is obtained for the new unknowns (i.e. equations (32), (C.22), (C.26) and (C.29) in appendix C):

$$
\begin{aligned}
& \mathcal{F}_{b c}^{ \pm}:=D_{b c} \Phi_{ \pm}=0 \quad R_{2}:=h^{a b} D_{a} \Phi_{+} D_{b} \Phi_{-}=0 \\
& \mathcal{E}_{a}:=\left(\mathbf{e}_{a} \psi\right)+\Omega_{b} \mathbf{e}_{a}^{b}+\mu_{c} \bar{\gamma}_{a b}^{c} \bar{h}^{b d} v_{d}=0 \\
& \mathcal{W}:=\left(\mathbf{v} \Omega_{2}\right)-\left(\mathbf{w} \Omega_{1}\right)+\bar{h}^{b c} \Omega_{b} \Omega_{c}+\frac{1}{2} \overline{\mathcal{R}}=0
\end{aligned}
$$




$$
\begin{aligned}
\mathcal{T}:=\zeta_{1}\left(\mathbf{w} \Omega_{1}\right) & -\zeta_{2}\left(\mathbf{v} \Omega_{2}\right)-\frac{1}{2}\left[\left(\mathbf{w}^{2} \zeta_{1}\right)+\left(\mathbf{v}^{2} \zeta_{2}\right)\right] \\
& -\left(\zeta_{1} \Omega_{1}^{2}+\zeta_{2} \Omega_{2}^{2}\right)+\frac{3}{2} \Omega_{1}\left(\mathbf{w} \zeta_{1}\right)-\frac{3}{2} \Omega_{2}\left(\mathbf{v} \zeta_{2}\right)=0,
\end{aligned}
$$

where $\mathbf{v}$ and $\mathbf{w}$ are related to a given $\bar{h}$-orthonormal frame $\left\{\mathbf{e}_{3}, \mathbf{e}_{4}\right\}$ by (C.18) and $\Omega_{b}, \zeta_{\alpha}$ ( $b=1,2, \alpha=1,2)$ are given in terms of the unknowns by equations (C.24), (C.14), (C.9) and (C.10).

The number of equations exceeding by far the number of unknowns are dealt much in the same way as it is usually done with Einstein equations: considering a certain subset of distinguished equations as the reduced PDS, and treating the remaining equations as constraints; the existence of solutions will then be studied as a Cauchy problem.

We choose a hypersurface $\Sigma$ in $\mathcal{S}$ which will act as a Cauchy hypersurface for the partial differential system $(\Sigma$ is actually a curve because $\operatorname{dim} \mathcal{S}=2)$, and take Gaussian $\bar{h}$-normal coordinates $\left(y^{1}, y^{2}\right)$ in a neighbourhood of $\Sigma$, so that $y^{2}=0$ on $\Sigma$ and

$\bar{h}_{12}=\bar{h}^{12}=0, \quad \bar{h}_{22}=\bar{h}^{22}=1, \quad \bar{h}_{11}=U \quad$ and $\quad \bar{h}^{11}=U^{-1}$.

Thus, the given $\bar{h}$-orthonormal may be taken to be

$$
\mathbf{e}_{1}=\frac{1}{\sqrt{U}} \partial_{1}, \quad \mathbf{e}_{2}=\partial_{2} \quad \text { and } \quad \alpha^{1}=\sqrt{U} \mathrm{~d} y^{1}, \quad \alpha^{2}=\mathrm{d} y^{2} .
$$

We can now consider (43)-(46) as a system of differential equations in the five unknowns $\Phi_{ \pm}, a, b$ and $\psi$, and separate:

the reduced system, namely

$$
\mathcal{F}_{22}^{ \pm}=0, \quad \mathcal{E}_{2}=0, \quad \mathcal{W}=0 \quad \text { and } \quad \mathcal{T}=0, \quad \text { and }
$$

\section{the constraints}

$$
\mathcal{F}_{1 a}^{ \pm}=0, \quad R_{2}=0 \quad \text { and } \quad \mathcal{E}_{1}=0,
$$

$(a=1,2)$ to be satisfied by the Cauchy data on $\Sigma$.

Proposition 1. Any solution of the reduced system (48) fulfilling constraints (49) on the Cauchy hypersurface $\Sigma$ also fulfils the constraints in an open neighbourhood of $\Sigma$.

Proof. By differentiating (43) and (44), we easily obtain

$$
\begin{array}{ll}
D_{[c} \mathcal{F}_{a] b}^{ \pm} & \equiv-\frac{\mathcal{R}}{2} D_{d} \Phi_{ \pm} \epsilon_{b}^{d} \epsilon_{c a}, \quad D_{a} R_{2} \equiv \frac{1}{2} h^{b c}\left(\mathcal{F}_{a b}^{+} D_{c} \Phi_{-}+\mathcal{F}_{a b}^{-} D_{c} \Phi_{+}\right) \quad \text { and } \\
\bar{D}_{[a} \mathcal{E}_{b]} \equiv \frac{\mathcal{W}}{2} \bar{\epsilon}_{a b} &
\end{array}
$$

and, particularly, since we are dealing with a solution of the reduced system (48), we obtain for the constraints

$$
D_{2} R_{2}=\frac{1}{2} U^{-1}\left(\mathcal{F}_{21}^{+} D_{1} \Phi_{-}+\mathcal{F}_{21}^{-} D_{1} \Phi_{+}\right), \quad D_{2} \mathcal{F}_{1 a}^{ \pm}=D_{1} \mathcal{F}_{21}^{ \pm} \delta_{a}^{1} \quad \text { and } \quad \bar{D}_{2} \mathcal{E}_{1}=0
$$

$a=1,2$, which is a linear, homogeneous, partial differential system to be fulfilled by the constraints, whence it follows that the vanishing of the constraints on $\Sigma$ propagates to an open neighbourhood of $\Sigma$. 


\subsection{The reduced system. Characteristic determinant}

To decide whether $\Sigma$ is a non-characteristic hypersurface for the reduced system (48), we must study its characteristic determinant [13]. To this end we must consider only the principal part of its equations, i.e. the part containing the highest order derivatives of the unknowns, particularly, $\partial_{2}^{2} \Phi_{ \pm}, \partial_{2}^{2} a, \partial_{2}^{2} b$ and $\partial_{2} \psi$, and we easily obtain that

$$
\mathcal{F}_{22}^{ \pm} \cong \partial_{2}^{2} \Phi_{ \pm}, \quad \mathcal{E}_{2} \cong \partial_{2} \psi
$$

where $\cong$ means 'equal apart from non-principal terms'.

The principal parts of the remaining two equations are not so simple; they look like

$$
\begin{aligned}
& \mathcal{W}=\mathcal{W}^{+} \partial_{2}^{2} \Phi_{+}+\mathcal{W}^{-} \partial_{2}^{2} \Phi_{-}+\mathcal{W}^{(\psi)} \partial_{2} \psi+\mathcal{W}^{(a)} \partial_{2}^{2} a+\mathcal{W}^{(b)} \partial_{2}^{2} b \\
& \mathcal{T}=\mathcal{T}^{+} \partial_{2}^{2} \Phi_{+}+\mathcal{T}^{-} \partial_{2}^{2} \Phi_{-}+\mathcal{T}^{(\psi)} \partial_{2} \psi+\mathcal{T}^{(a)} \partial_{2}^{2} a+\mathcal{T}^{(b)} \partial_{2}^{2} b .
\end{aligned}
$$

It easily follows that the characteristic determinant of the reduced system is $\chi=\mathcal{W}^{(a)} \mathcal{T}^{(b)}-$ $\mathcal{W}^{(b)} \mathcal{T}^{(a)}$ and we do not need to calculate explicitly all the coefficients in the principal part of $\mathcal{W}$ and $\mathcal{T}$. A detailed, heavy-going calculation yields

$\chi=\frac{b \sin 2 \psi}{16 x_{1} x_{2}}\left[\sin 2 \psi \frac{\left(Z_{1}-Z_{2}\right)^{2}}{a(a+b)}-\left(x_{1}+x_{2}\right)\left(Z_{1}+Z_{2}+\left(Z_{1}-Z_{2}\right) \cos 2 \psi\right)\right]$,

where $x_{\alpha}$ and $Z_{\alpha}$ are given in appendix C. Then, in order that $\Sigma$ is a non-characteristic hypersurface, Cauchy data must be chosen so that $\chi \neq 0$.

\subsection{The constraints}

The Cauchy data, namely $\Phi_{ \pm}, \dot{\Phi}_{ \pm}:=\partial_{4} \Phi_{ \pm}, a, b, \dot{a}, \dot{b}$ and $\psi$ on $\Sigma$, must be chosen so that $\chi \neq 0$ and constraints (49) are fulfilled. $\Sigma$ is a curve and the coordinate $u:=y^{3}$ acts as a curve parameter; the constraints can thus be written as

$$
\left.\begin{array}{l}
\mathcal{F}_{12}^{ \pm}:=\frac{\mathrm{d} \dot{\Phi}_{ \pm}}{\mathrm{d} u}-\Gamma_{12}^{c} D_{c} \Phi_{ \pm}=0 \\
\mathcal{F}_{11}^{ \pm}:=\frac{\mathrm{d}^{2} \Phi_{ \pm}}{\mathrm{d} u^{2}}-\Gamma_{11}^{c} D_{c} \Phi_{ \pm}=0 \\
\mathcal{E}_{1}:=\frac{\mathrm{d} \psi}{\mathrm{d} u}-\bar{\Gamma}_{11}^{4} \frac{1}{\sqrt{U}}+\Omega_{1}=0
\end{array}\right\}
$$

$(c=1,2)$, and we must replace $D_{1} \Phi_{ \pm}$by $\frac{\mathrm{d} \Phi_{ \pm}}{\mathrm{d} u}, D_{2} \Phi_{ \pm}$by $\dot{\Phi}_{ \pm}$and so on. $\Gamma_{e d}^{c}$ and $\bar{\Gamma}_{e d}^{c}$ are the Christoffel symbols for the connections $D$ and $\bar{D}$, respectively, and they depend on $\Phi_{ \pm}, a$, $b$, their first-order derivatives and $\psi$.

We can therefore prescribe arbitrary values for $a, \dot{a}, b$ and $\dot{b}$ on $\Sigma$, because there is no constraint on them, and then substitute them into (51) which can be taken as an ordinary differential system on the remaining Cauchy data: $\Phi_{ \pm}, \dot{\Phi}_{ \pm}$and $\psi$ on the curve $\Sigma$. This system admits a solution for any given initial data $\Phi_{ \pm}\left(x_{0}\right), D_{1} \Phi_{ \pm}\left(x_{0}\right)$ and $\psi\left(x_{0}\right)$, for a given point $x_{0} \in \Sigma$.

As for the remaining constraint, $R_{2}:=\frac{1}{2} h^{b c} D_{b} \Phi_{+} D_{c} \Phi_{-}=0$, it acts merely as a condition on the initial data $D_{c} \Phi_{ \pm}\left(x_{0}\right)$.

\subsection{Summary of the proof}

We now show how to construct the deformed metric $\eta_{a b}$ from a solution to the above Cauchy problem. 
(1) Take an analytic curve $\Sigma_{0} \subset \mathcal{S}$ and choose a point $x_{0} \in \Sigma_{0}$.

(2) Choose constant $\hat{m}_{A}$ and $\hat{n}_{A}$ such that $\hat{m}_{1} \hat{n}_{2}-\hat{n}_{1} \hat{m}_{2}=1$.

(3) Give $a, \dot{a}, b$ and $\dot{b}$, analytic functions on $\Sigma_{0}$, then

(4) give $\psi\left(x_{0}\right), \Phi_{ \pm}\left(x_{0}\right)$ and $D_{c} \Phi_{ \pm}\left(x_{0}\right)(c=3,4)$.

(5) Then solve the ordinary differential system (51) to obtain $\psi, \Phi_{ \pm}$and $\dot{\Phi}_{ \pm}$on $\Sigma$, a neighbourhood of $x_{0}$ in $\Sigma_{0}$.

(6) With these $a, b, \dot{a}, \dot{b}, \Phi_{ \pm}, \dot{\Phi}_{ \pm}$and $\psi$ as Cauchy data on $\Sigma$, the reduced system has an analytic solution,

$$
a, \quad b, \quad \Phi_{ \pm}, \quad \psi, \quad \text { on a neighbourhood of } \quad \Sigma .
$$

(7) With these five functions obtain $h_{a b}, \kappa_{a}^{A}$ and $\lambda_{A B}$, as indicated in appendix C.1 and equation (31).

(8) Finally, taking $\xi_{a}^{A}=\bar{\xi}_{a}^{A}+\kappa_{a}^{A}$ and using (36), the flat metric $\eta_{a b}$ is obtained.

\section{A particularly simple case}

We shall now consider a case when both almost-product structures, one defined by the elliptic 2-projector $H_{a b}$ in the deformation law (34) and another one by the orbits of the Killing vectors, are the same. The original and deformed metrics respectively are

$$
g_{a b}=\bar{h}_{a b}+\bar{k}_{a b} \quad \text { and } \quad \eta_{a b}=\varphi \bar{h}_{a b}+a \bar{k}_{a b}
$$

with $\bar{k}_{a b}:=\bar{\lambda}_{A B} \bar{\xi}_{a}^{A} \bar{\xi}_{b}^{B}$ and $\varphi:=a+b$. In this case, which we shall refer to as a degenerate deformation law, both metrics are related by a biconformal transformation.

This is indeed a non-generic case: a metric $g_{a b}$ with two commuting Killing vectors does not, in general, admit a degenerate deformation law yielding a flat $\eta_{a b}$. We shall here characterize the metrics $g_{a b}$ admitting a degenerate deformation.

From (52) we have that

$$
\begin{aligned}
& \xi_{A a}=a \bar{\xi}_{A a}, \quad \lambda_{A B}=a \bar{\lambda}_{A B}, \quad \lambda^{A B}=\frac{1}{a} \bar{\lambda}^{A B} \\
& \xi_{b}^{A}=\bar{\xi}_{b}^{A} \quad \text { and } \quad h_{a b}=\varphi \bar{h}_{a b},
\end{aligned}
$$

whence it follows that

$$
\tau=a \bar{\tau}, \quad \bar{\theta}^{A}=\varphi \theta^{A} \quad \text { and } \quad \bar{\theta}_{A}=\theta_{A} \varphi / a
$$

where the fact that $\epsilon_{a b}=\varphi \bar{\epsilon}_{a b}$ has been included.

Since $\eta_{a b}$ is flat and has two commuting Killing vectors (see appendix B), only two possibilities are left:

(a) $\theta_{1}=\theta_{2}=0$ which, by (54), implies that $\bar{\theta}_{1}=\bar{\theta}_{2}=0$ and

(b) $\theta_{A}=k_{A} \theta$, with $k_{A}=$ constant, which implies that $\bar{\theta}_{A}=k_{A} \bar{\theta}$.

Note that $\tilde{\bar{\lambda}}_{A B}:=\bar{\tau}^{-1} \bar{\lambda}_{A B} \sqrt{2}=\tau^{-1} \lambda_{A B} \sqrt{2}=\tilde{\lambda}_{A B}$ and, as this $\tilde{\lambda}_{A B}$ corresponds to the metric $\eta_{a b}$ which is flat, the results derived in appendix B apply. Particularly from (B.8) we have that

$$
\mathrm{d} \tilde{\bar{\lambda}}_{A B}=\mathrm{d} \tilde{\lambda}_{A B}=q_{A B}(f) \mathrm{d} f,
$$

where $q_{A B}(f)$ is derived from $\mathrm{d} \tilde{\bar{\lambda}}_{A B}$ as indicated in proposition 3

Case (a). From proposition 3 in appendix B and equations (22) and (55), we have that

$$
\bar{R}_{3}=0
$$

6 These data must be chosen so that the characteristic determinant does not vanish at $x_{0}$. 
which is a necessary condition to be fulfilled by $g_{a b}$ in order to admit a degenerate deformation law. Thus, we must first check whether $\bar{R}_{3}=0$ and then take $q=0$ if $\operatorname{det}\left(q_{A B}\right)=0$ or $q=\operatorname{sign}\left(\operatorname{det}\left(q_{A B}\right)\right)$ otherwise.

(a1) If $q=0$, we have that (equation (30)) $\tilde{\bar{\lambda}}_{A B}=\tilde{\lambda}_{A B}=\hat{\lambda}_{A B}+\hat{q}_{A B} F$, with $\hat{\lambda}_{A B}$ and $\hat{q}_{A B}$ constant, $\hat{\lambda}^{A B} \hat{q}_{A B}=0$ and $\operatorname{det}\left(\hat{\lambda}_{A B}=-1\right.$. Hence, from $\mathrm{d} \tilde{\bar{\lambda}}_{A B}=\hat{q}_{A B} \mathrm{~d} F$ it is immediate to determine $\hat{q}_{A B}$ and $\mathrm{d} F$ (apart form a constant factor).

Now, by (30) we also have that $\tau=\tau_{0}$ constant and $D_{b} F_{c}=0$, which leads to

$$
a=\tau_{0} / \bar{\tau} \quad \text { and } \quad \bar{D}_{b} F_{c}-\psi_{(b} F_{c)}+\frac{1}{2}\left(\psi_{e} F_{a} \bar{h}^{a e}\right) \bar{h}_{b c}=0,
$$

where $\psi:=\log \varphi$ and the relation between both connections, $D$ and $\bar{D}$, has been included.

On the one hand, the second equation implies that

$$
\bar{h}^{b c} \bar{D}_{b} F_{c}=0
$$

which is a constraint on $F$ and, on the other, it allows us to obtain

$$
\psi_{b}=\bar{D}_{b} \log \|F\|^{2}, \quad \text { where } \quad \log \|F\|^{2}:=F_{e} F_{a} \bar{h}^{a e},
$$

that is, $\psi=\log \|F\|^{2}+$ constant.

Combining now this equation with (20) and including that $h_{a b}=\mathrm{e}^{\psi} \bar{h}_{a b}$, we arrive at [11]

$$
\overline{\mathcal{R}}+\bar{D}^{b} \bar{D}_{b} \log \|F\|^{2}=0,
$$

which is a further necessary condition connecting $F$ and $\overline{\mathcal{R}}$.

(a2) If $q=-1$ (see appendix B, right after (B.16)), then $\lambda_{A B}$ must be constant and this implies that $a \in \Lambda^{0} \mathcal{S}$ must exist such that $a \bar{\lambda}_{A B}=\lambda_{A B}=$ constant. In this case equation (20) becomes a condition on the conformal factor $\varphi=\mathrm{e}^{\psi}$, namely [11]

$$
\overline{\mathcal{R}}+\bar{h}^{b c} \bar{D}_{b c} \psi=0
$$

(a3) If $q=+1$, define

$$
\mathrm{d} f:=\sqrt{\operatorname{det}\left(\tilde{\mathrm{d}}_{A B}\right)} \quad \text { and } \quad q_{a b}:=\frac{\mathrm{d} \tilde{\bar{\lambda}}_{A B}}{\mathrm{~d} f} .
$$

Then, using (B.21), (31) and the fact that $\tilde{\bar{\lambda}}_{A B}=\tilde{\lambda}_{A B}$, we obtain

$$
\tilde{\bar{\lambda}}_{A B}+q_{A B}=\mathrm{e}^{f} \hat{n}_{A} \hat{n}_{B} \quad \text { and } \quad-\tilde{\bar{\lambda}}_{A B}+q_{A B}=\mathrm{e}^{-f} \hat{m}_{A} \hat{m}_{B}
$$

where $\hat{m}_{A}, \hat{n}_{A}$ are constant and $\hat{m}_{2} \hat{n}_{1}-\hat{m}_{1} \hat{n}_{2}=1$. This is a necessary condition to be fulfilled by $\bar{\lambda}_{A B}$ which will ensure that equation (25) is satisfied and will allow to derive $f, \hat{m}_{A}$ and $\hat{n}_{A}$.

The functions $f$ and $t=\log \tau$ must fulfil (B.19) and $R_{2}=0$, which respectively amount to

$$
\begin{aligned}
& \bar{D}^{b} f_{b}+\bar{h}^{b c} t_{b} f_{c}=0, \\
& \bar{D}_{b}\left[t+\log \|f\|^{2}-\psi\right]-\frac{1}{\|f\|^{2}}\left(\bar{D}^{a} f_{a}\right) f_{b}=0 \\
& \bar{D}^{b} t_{b}+\|t\|^{2}=0, \\
& \bar{D}_{b}\left[t+\log \|t\|^{2}-\psi\right]-\frac{1}{\|t\|^{2}}\left(\bar{h}^{a c} t_{a} f_{c}\right) f_{b}=0 \quad \text { and }
\end{aligned}
$$




$$
\|t\|^{2}=\|f\|^{2} \quad \text { where } \quad\|t\|^{2}:=\bar{h}^{a c} t_{a} t_{c} .
$$

Furthermore, condition (20) implies that (see [11]) $\quad \overline{\mathcal{R}}+\bar{h}^{b c} \bar{D}_{c} \psi_{b}=0$, or

$$
\overline{\mathcal{R}}+\bar{D}^{b} t_{b}+\bar{D}^{b} \bar{D}_{c} \log \|t\|^{2}+\bar{D}^{b}\left(\frac{\bar{h}^{a e} t_{a} f_{e}}{\|t\|^{2}} f_{b}\right)=0 .
$$

Since $f$ is known, equations (63) and (67) allow us to determine

$$
t_{b}=-\frac{\left(\bar{D}^{a} f_{a}\right)}{\|f\|^{2}} f_{b} \pm \frac{\sqrt{\|f\|^{4}-\left(\bar{D}^{a} f_{a}\right)^{2}}}{\|f\|^{2}} \epsilon_{b}^{c} f_{c} .
$$

On its turn, equation (66) is a combination of (63), (64) and (67); equation (64) yields $\psi_{b}$ and the remaining two equations, i.e. (65) and (68), imply conditions to be fulfilled by $f$, respectively,

$-\bar{D}^{b}\left(\frac{\left(\bar{D}^{a} f_{a}\right)}{\|f\|^{2}} f_{b}\right) \pm \bar{D}^{b}\left(\frac{\sqrt{\|f\|^{4}-\left(\bar{D}^{a} f_{a}\right)^{2}}}{\|f\|^{2}}\right) \epsilon_{b}^{c} f_{c}+\|f\|^{2}=0$

and

$$
\overline{\mathcal{R}}-\|f\|^{2}+\bar{D}^{b} \bar{D}_{c} \log \|f\|^{2}-\bar{D}^{b}\left(\frac{\left(\bar{D}^{a} f_{a}\right)}{\|f\|^{2}} f_{b}\right)=0
$$

Case (b). If the twists $\bar{\theta}_{A}$ do not vanish we are compelled to try with case (b) and equation (54) imposes a first restriction, namely

$$
\text { a couple of constants }\left(k_{1}, k_{2}\right) \neq(0,0) \text { must exist such that } \quad \bar{\theta}_{A}=k_{A} \bar{\theta} \text {. }
$$

Furthermore, from proposition 2 in appendix B we have that $\lambda \in \Lambda^{0} \mathcal{S}$ and $\hat{\lambda}_{A B}$ constant must exist such that $\lambda_{A B}=k_{A} k_{B} \lambda+\hat{\lambda}_{A B}$. Taking in consideration equation (53), this is equivalent to

$\exists a \in \Lambda^{0} \mathcal{S} \quad$ such that $a \bar{\lambda}_{A B} l^{B}=p_{A}$ constant, $\quad$ with $\quad l^{A}=\left(k_{2},-k_{1}\right)$

(as $\lambda_{A B}$ is non-degenerated, $\left(p_{1}, p_{2}\right) \neq(0,0)$ ), which in turn is equivalent to

$$
\exists q^{A}:=\left(p_{2},-p_{1}\right) \quad \text { such that } \quad \bar{\lambda}_{A B} q^{A} l^{B}=0 .
$$

If this happens, the factor $a \in \Lambda^{0} \mathcal{S}$ is

$$
a=\frac{p_{1}^{2}+p_{2}^{2}}{\bar{\lambda}_{A B} p^{A} l^{B}} \quad \text { with } \quad p^{A}:=\left(p_{1}, p_{2}\right) .
$$

Now, from (B.1) and (54) we have that

$$
\varphi=\frac{a^{2} \bar{\theta} \bar{\tau}}{C}, \quad \text { with } \quad C=\text { constant. }
$$

This factor must furthermore fulfil the additional conditions implied by (B.5) and (B.6) that, after some algebra, yield [11]

$$
\begin{aligned}
& \bar{D}^{b} \psi_{b}+\overline{\mathcal{R}}+\frac{3 \alpha C \bar{\theta} a}{\tau^{3}}, \quad \bar{D}^{b} \tau_{b}=\frac{\alpha C \bar{\theta} a}{\tau^{2}} \\
& \text { and } \quad \psi_{b}=\bar{D}_{b} \log \|\tau\|^{2}+\tau_{b} \frac{\alpha C^{2} \varphi}{\tau^{3}\|\tau\|^{2}},
\end{aligned}
$$

where $\psi:=\log \varphi, \tau=a \bar{\tau}$ and $\|\tau\|^{2}:=\tau_{b} \tau_{c} \bar{h}^{b c}$.

Summarizing, if the twists $\bar{\theta}_{A}$ do not vanish, we must first check whether (72) and (74) are fulfilled; then compute $a$ and $\varphi$ defined by (75) and check if relations (76) and (77) hold. 


\subsection{Example: stationary axisymmetric spacetimes}

We now consider the case of a stationary axisymmetric spacetime [12] whose line element is

$$
\mathrm{d} s^{2}=\mathrm{e}^{-2 U+2 K}\left(\mathrm{~d} \rho^{2}+\mathrm{d} z^{2}\right)+\mathrm{e}^{-2 U} \rho^{2} \mathrm{~d} \phi^{2}-\mathrm{e}^{2 U}(\mathrm{~d} t+N \mathrm{~d} \phi)^{2},
$$

where $N, U$ and $K$ are arbitrary functions of $\rho$ and $z$. The Killing vectors are $X_{1}=\partial_{\phi}$ and $X_{2}=\partial_{t}$ and the associated 1-forms are

$$
\bar{\xi}_{1}=N \bar{\xi}_{2}+\mathrm{e}^{-2 U} \rho^{2} \mathrm{~d} \phi \quad \text { and } \quad \bar{\xi}_{2}=-\mathrm{e}^{2 U}(\mathrm{~d} t+N \mathrm{~d} \phi) .
$$

Therefore, we have that $\bar{h}_{b c}=\mathrm{e}^{-2 U+2 K} \delta_{b c}$ and

$\bar{\lambda}_{A B}=\mathrm{e}^{-2 U} \rho^{2} \delta_{A}^{1} \delta_{B}^{1}-\mathrm{e}^{2 U} u_{A} u_{B}, \quad$ with $\quad u_{1}=N, \quad u_{2}=1$,

the determinant is $\bar{\tau}=\sqrt{2} \rho$ and the inverse matrix is

$\bar{\lambda}^{A B}=-\mathrm{e}^{-2 U} \delta_{2}^{A} \delta_{2}^{B}+\frac{\mathrm{e}^{2 U}}{\rho^{2}} v^{A} v^{B}, \quad$ with $\quad v^{1}=1, \quad v^{2}=-N$.

It can be easily checked that

$$
\bar{R}_{3}=0 \quad \Leftrightarrow \quad \bar{\lambda}^{A B} \mathrm{~d} \bar{\lambda}_{1 A} \wedge \mathrm{d} \bar{\lambda}_{2 B}=0 \quad \Leftrightarrow \quad \mathrm{e}^{-2 U} \rho=L(N),
$$

where $L(N)$ is an arbitrary function of the variable $N$.

Then, by differentiating $\tilde{\bar{\lambda}}_{A B}:=\bar{\lambda}_{A B} \sqrt{2} / \bar{\tau}$ we obtain

$$
\mathrm{d} \tilde{\bar{\lambda}}_{A B}=Q_{A B} \mathrm{~d} N \quad \text { with } \quad Q_{A B}=L^{\prime} \delta_{A}^{1} \delta_{B}^{1}+\frac{L^{\prime}}{L^{2}} u_{A} u_{B}-\frac{2}{L} \delta_{(A}^{1} u_{B)} .
$$

Now, let $Q:=\operatorname{det}\left(Q_{A B}\right)=\left(L^{\prime 2}-1\right) / L^{2}$. If $Q \neq 0$, we must take $\mathrm{d} f:=\mathrm{d} N \sqrt{|Q|}$ and $q_{A B}:=Q_{A B} / \sqrt{|Q|}$ and, by (B.20) we have that

$$
\frac{\mathrm{d} Q_{A B}}{\mathrm{~d} N}-\frac{1}{2 Q} \frac{\mathrm{d} Q}{\mathrm{~d} N} Q_{A B}-|Q| \tilde{\bar{\lambda}}_{A B}=0 .
$$

This has a solution only in case that $L^{\prime}=0$, which contradicts the initial assumption that $Q \neq 0$.

If $Q=0$, by conveniently choosing the sign of $\phi$ we get $L^{\prime}=1$ or $L=N+C$ with $C=$ constant. Then, the case $q=0$ in appendix B applies and, from equation (B.18), we have that $\tilde{\bar{\lambda}}_{A B}=\hat{\lambda}_{A B}+F \hat{q}_{A B}$ with

$$
\hat{\lambda}_{A B}=\left(\begin{array}{cc}
2 C & -1 \\
-1 & 0
\end{array}\right), \quad \hat{q}_{A B}=\left(\begin{array}{cc}
C^{2} & -C \\
-C & 1
\end{array}\right)
$$

and $F=-L^{-1}$. The results for case (a.1) in section 2.2 also apply and we have that

$a=\tau_{0} / \bar{\tau}, \quad \psi=6 U-2 K+\log H, \quad \delta^{b c} \partial_{b c}\left(\mathrm{e}^{2 U} / \rho\right)=0$

with $H:=\rho^{-4} \delta^{b c}\left(2 \rho U_{b}-\delta_{b}^{1}\right)\left(2 \rho U_{c}-\delta_{c}^{1}\right)$.

Besides, we must take into consideration that $h_{b c}:=\mathrm{e}^{\psi} \bar{h}_{b c}=\mathrm{e}^{\psi-2 U+2 K} \delta_{b c}$ is flat, which is equivalent to [11]

$$
\delta^{b c} \partial_{b c}(4 U+\log H)=0 .
$$

Summarizing, a degenerated deformation law exists that transforms the stationary axisymmetric metric (78) into a flat metric iff (i) $\bar{R}_{3}=0$, (ii) a constant $C$ exists such that $\rho \mathrm{e}^{-2 U}=N+C$, (iii) $\bar{\lambda}_{A B} / \rho$ has the form (82) and (iv) $U$ simultaneously fulfils (83) and (84). In such a case, the biconformal factors are $a=\tau_{0} / \bar{\tau}$ and $\varphi=\mathrm{e}^{\psi}$ with $\psi$ given by (83). 


\section{Concluding remarks}

We have studied the structure of the Riemann tensor for a spacetime admitting two commuting Killing vectors and its expression in terms of the horizontal (quotient) metric, the vertical metric (on the orbits) and the kinematic invariants of the orbits as spacetime submanifolds. We have applied these results to find a solution to the reconstruction problem, particularly to obtain a flat Lorentzian metric given two commuting Killing fields and the quotient metric.

The central result of the paper is the proof of theorem 1, namely, given a spacetime admitting two commuting Killing vectors, there exists a biconformal deformation law (34) which transforms the spacetime metric $g_{a b}$ into a flat metric $\eta_{a b}$ admitting the same Killing vectors as $g_{a b}$.

The proof is based on an application of the Cauchy-Kovalewski theorem and, due to the arbitrariness in the choice of the Cauchy hypersurface and Cauchy data, the solution is not unique - as it happens for an analogous existence theorem in the general case [1], with no Killing vectors. The present result actually intends to find a way to reduce this lack of uniqueness.

The aforementioned biconformal deformation law is carried out following the $2+2$ almostproduct structure associated with the 2-projector $H_{b}^{a}$. In general, this structure does not coincide with the almost-product structure associated with the Killing orbits. When both structures coincide we refer to as degenerated deformation law. We have also studied what conditions must the background spacetime fulfil in order that there exists a degenerated law deforming the spacetime metric to a flat metric. We have finally particularized the obtained conditions to a stationary axisymmetric spacetime.

We conjecture that this result may be extended to the more general case of $g_{a b}$ admitting a Killing algebra $\mathcal{G}$ which is a subalgebra of the Poincare algebra $\mathcal{P}$. Although at this point we cannot provide a proof to this statement, some ongoing developments considering dimensionality and subalgebra structure of $\mathcal{G}$ strongly suggest this to be the case.

\section{Acknowledgments}

JC acknowledges financial support from the Spanish Ministerio de Educación y Ciencia through grant no FPA-2007-60220. Partial financial support from the Govern de les Illes Balears is also acknowledged. JL acknowledges financial support from Ministerio de Educación y Ciencia through grant no FIS2007-63034 and from the Generalitat de Catalunya, 2009SGR-417 (DURSI).

\section{Appendix A. Some bivectors and derivatives}

The following bivectors and bivector equalities will be useful:

$$
\Omega_{a b}:=\frac{\tau}{2} \xi_{a}^{1} \wedge \xi_{b}^{2}=\frac{\tau}{2} \sigma_{A B} \xi_{a}^{A} \xi_{b}^{B}=\frac{1}{\tau} \sigma^{C D} \xi_{C a} \xi_{D b}=-\frac{1}{\tau} \xi_{1 a} \wedge \xi_{2 b}
$$

where $\sigma_{A B}$ is skewsymmetric and $\sigma_{12}=1$. It is obvious that

$$
\Omega_{a c} \Omega^{a b}=-\frac{1}{2} \xi_{c}^{B} \xi_{B}^{b} \quad \text { and } \quad \sigma^{A B}=\lambda^{A C} \lambda^{B D} \sigma_{C D} \frac{\tau^{2}}{2}, \quad \sigma^{A B} \sigma_{B C}=\delta_{C}^{A},
$$

where $\sigma^{12}=-1$, and that $\mathcal{L}_{X_{A}} \Omega_{a b}=0$.

The volume tensor on $\mathcal{S}$ :

$$
\epsilon_{a b}:=\frac{\sqrt{2}}{\tau} \epsilon_{a b c d} X_{1}^{c} X_{2}^{d}=-\frac{1}{\tau \sqrt{2}} \sigma^{C D} \epsilon_{a b c d} X_{C}^{c} X_{D}^{d}
$$

and $\epsilon^{a b} \epsilon_{c b}=h_{c}^{a}$. It is obvious that $\epsilon_{c b}$ is horizontal and Lie-constant; hence $\epsilon_{c b} \in \Lambda^{2} \mathcal{S}$. 
The dual bivectors respectively are

$$
\tilde{\epsilon}_{e f}=\sqrt{2} \Omega_{e f}, \quad \tilde{\Omega}_{e f}=-\frac{1}{\sqrt{2}} \epsilon_{e f} .
$$

Furthermore, if $w_{a}$ is a vector on $\mathcal{S}$, then

$$
\left(\xi^{A} \wedge w\right)_{e f}^{\sim}=-\frac{2 \sqrt{2}}{\tau} \sigma^{A B} \xi_{B[e} \epsilon_{f] c} w^{c} .
$$

If $w^{b}$ is a vector field on $\mathcal{S}$, from $\left[X_{A}, w\right]=0$, it follows that

$$
\nabla_{A} w^{b}=w^{d}\left(\frac{1}{2} \lambda_{A B \mid d} \xi^{B b}+\frac{1}{2} \theta_{A} \epsilon_{d}^{b}\right)
$$

and, also,

$\nabla_{A} T^{b c}=T^{d c}\left(\frac{1}{2} \lambda_{A B \mid d} \xi^{B b}+\frac{1}{2} \theta_{A} \epsilon_{d}^{b}\right)+T^{b d}\left(\frac{1}{2} \lambda_{A B \mid d} \xi^{B c}+\frac{1}{2} \theta_{A} \epsilon_{d}^{c}\right)$

where $\nabla_{A}$ stands for $X_{A}^{a} \nabla_{a}$. Now, using the identity: $\mathrm{d}\left(\log \left|\operatorname{det} \lambda_{A B}\right|\right)=\mathrm{d} \lambda_{A B} \lambda^{A B}$, from (4) we have

$$
t_{a}=\frac{1}{2} \lambda^{A B} \lambda_{A B \mid a}, \quad t:=\log \tau .
$$

\section{Appendix B.}

Our goal here is to see how equations (28), namely

$$
R_{1}=R_{2}=R_{3}, \quad P_{A b}=Q_{A b}=0, \quad P_{D c A b}=0
$$

constrain the possible values of $\lambda_{A B}, \kappa_{a}^{A}$ and $h_{a b}$.

Proposition 2. If $P_{A b}=Q_{A b}=0$, then

(a) either $\theta_{1}=\theta_{2}=0$ or

(b) two constants, $k_{A}$, exist such that $\theta_{A}=k_{A} \theta$ and $\mathrm{d} \lambda_{A B}=k_{A} k_{B} \mathrm{~d} \lambda$, where $\theta, \lambda \in \Lambda^{0}(\mathcal{S})$. In this case one also has

$$
\theta \tau=C \quad \text { and } \quad-\frac{\tau^{2}}{2}=\alpha \lambda+\hat{\delta}
$$

with $C, \alpha$ and $\hat{\delta}$ constant.

Proof. Indeed, by equation (23), $P_{A b}=0$ implies $2 \mathrm{~d} \theta_{A}+\theta_{C} \lambda^{C T} \mathrm{~d} \lambda_{T A}=0$. Multiplying it by $\theta_{A^{\prime}}, A^{\prime} \neq A$, and using that $Q_{T b}=0$ amounts to $\theta_{1} \mathrm{~d} \lambda_{T 2}=\theta_{2} \mathrm{~d} \lambda_{T 1}$, one readily obtains that $\theta_{1} \mathrm{~d} \theta_{2}=\theta_{2} \mathrm{~d} \theta_{1}$, which implies

(a) either $\theta_{1}=\theta_{2}=0$ or

(b) two constants $k_{A}$ exist such that $\theta_{A}=k_{A} \theta$, with $\theta \in \Lambda^{0}(\mathcal{S})$.

Furthermore, substituting this into $\theta_{1} \mathrm{~d} \lambda_{A 2}=\theta_{2} \mathrm{~d} \lambda_{A 1}$ and taking the symmetry of $\lambda_{A B}$ into account, we obtain that $\mathrm{d} \lambda_{A B}=k_{A} k_{B} \mathrm{~d} \lambda$, with $\lambda \in \Lambda^{0} \mathcal{S}$, and therefore

$$
\lambda_{A B}=k_{A} k_{B} \lambda+\hat{\lambda}_{A B} \quad \text { with } \quad \hat{\lambda}_{A B}=\hat{\lambda}_{B A}=\text { constant. }
$$

The inverse matrix $\lambda^{A B}$ is

$$
\lambda^{A B}=\frac{-2}{\tau^{2}}\left(l^{A} l^{B} \lambda+\hat{\lambda}^{A B}\right),
$$

where

$$
l^{A}=\left(k_{2},-k_{1}\right), \quad \hat{\lambda}^{A B}=\left(\begin{array}{cc}
\hat{\lambda}_{22} & -\hat{\lambda}_{12} \\
-\hat{\lambda}_{12} & \hat{\lambda}_{11}
\end{array}\right)
$$


and we also have that

$$
-\frac{\tau^{2}}{2}=\operatorname{det}\left(\lambda_{A B}\right)=\alpha \lambda+\hat{\delta}, \quad \text { with } \quad \alpha=\hat{\lambda}^{A B} k_{A} k_{B}, \quad \hat{\delta}:=\operatorname{det}\left(\hat{\lambda}_{A B}\right) .
$$

Substituting these into $P_{A b}=0$, we obtain $2 \mathrm{~d} \theta+\theta k_{C} \lambda^{C T} k_{T} \mathrm{~d} \lambda=0$, which implies that $\mathrm{d}\left(\theta\left|\operatorname{det}\left(\lambda_{A B}\right)\right|^{1 / 2}\right)=0 \quad$ or $\quad \theta \tau=C$, constant, where equation (4) has been included.

Let us now study the implications of the remaining curvature equations, $R_{1}=R_{2}=R_{3}=$ 0 and $P_{D c A b}=0$. Consider first the case (b): $\theta_{A}=k_{A} \theta$ and $\mathrm{d} \lambda_{A B}=k_{A} k_{B} \mathrm{~d} \lambda$. Equations $R_{2}=0$ and $R_{3}=0$ are identically satisfied and do not imply any further condition on $\theta, \tau$ or $k_{A}$. Then, taking (B.3) and (B.1) into account, equation $R_{1}=0$ implies that

$$
\mathcal{R}=-\frac{3 \alpha C^{2}}{\tau^{4}}
$$

and equation $P_{D c A b}=0$ amounts to

$$
D_{b c} \tau+\frac{\alpha C^{2}}{2 \tau^{3}} h_{b c}=0 .
$$

This is a partial differential system that is integrable provided that the Ricci scalar is (B.5). Combining now equations (B.5) and (B.6) we arrive at

$$
D_{b c} \mathcal{R}^{-1 / 4}+\frac{1}{6} \mathcal{R}^{3 / 4} h_{b c}=0
$$

which is a condition to be fulfilled by $\mathcal{R}$, the Ricci scalar of the given metric $h_{a b}$ on $\mathcal{S}$, in order that the ambient flat metric $\eta_{a b}$ exists.

Consider now the case (a): $\theta_{B}=0, B=1,2$, i.e. orthogonal transitivity, with no restriction on $\lambda_{A B}$. To start with, by (20), equation $R_{1}=0$ amounts to $\mathcal{R}=0$, which implies that $h_{a b}$ is flat.

Proposition 3. The scalar $R_{3}$ vanishes if, and only if, a function $f \in \Lambda^{0} \mathcal{S}$ and functions $q_{A B}(f)$ exist such that

$$
\mathrm{d}\left(\sqrt{2} \tau^{-1} \lambda_{A B}\right)=q_{A B}(f) \mathrm{d} f, \quad \text { where } \quad \lambda^{A B} q_{A B}=0,
$$

and $q:=\operatorname{det}\left(q_{A B}\right) \in\{0, \pm 1\}$.

Proof. Define $\tilde{\lambda}_{A B}:=\sqrt{2} \tau^{-1} \lambda_{A B}$. It is obvious from (4) that $\operatorname{det}\left(\tilde{\lambda}_{A B}\right)=-1$. From (22) we have that $R_{3}=2^{-3 / 2} \epsilon^{b c} \tilde{\lambda}^{T E} \tilde{\lambda}_{1 E \mid c} \tilde{\lambda}_{2 T \mid b}$, where $\tilde{\lambda}^{A B} \tilde{\lambda}_{B C}=\delta_{C}^{A}$, and therefore,

$$
R_{3}=0 \quad \Leftrightarrow \quad \tilde{\lambda}^{E T} \mathrm{~d} \tilde{\lambda}_{1 E} \wedge \mathrm{d} \tilde{\lambda}_{2 T}=0 .
$$

A short calculation then proves that this is equivalent to the existence of $F \in \Lambda^{1} \mathcal{S}$ such that $\mathrm{d} \tilde{\lambda}_{A B} \propto F$. Now, since $\operatorname{dim} \mathcal{S}=2, F$ is integrable, i.e. proportional to $\mathrm{d} u$ for some $u \in \Lambda^{0} \mathcal{S}$, whence it follows that $\mathrm{d} \tilde{\lambda}_{A B}=Q_{A B} \mathrm{~d} u$ for some $Q_{A B} \in \Lambda^{0} \mathcal{S}$ and the integrability conditions imply that $Q_{A B}=Q_{A B}(u)$.

Then (B.8) follows taking $f=u$ and $q_{A B}=Q_{A B}$, if $Q(u):=\operatorname{det}\left(Q_{A B}\right)=0$, or taking $\mathrm{d} f=\sqrt{|Q|} \mathrm{d} u$ and $q_{A B}=Q_{A B} / \sqrt{|Q|}$, if $Q(u) \neq 0$. Furthermore, $\operatorname{det}\left(\tilde{\lambda}_{A B}\right)=-1$ implies that $\tilde{\lambda}^{A B} \mathrm{~d} \tilde{\lambda}_{A B}=0$ or $\tilde{\lambda}^{A B} q_{A B}=0$.

Note that neither $f$ nor $q_{A B}$ vanishes except in the trivial case $\tilde{\lambda}_{A B}=$ constant.

$q_{A B}$ is a symmetric, traceless, 2-square matrix of functions on $\mathcal{S}$. Since the number of dimensions is 2 , using the characteristic polynomial we have that

$$
q_{A B} \tilde{\lambda}^{B C} q_{C D}=q \tilde{\lambda}_{A D}, \quad q:=\operatorname{det}\left(q_{A B}\right) \in\{0, \pm 1\} .
$$


Consider now the following quadratic form on the space of symmetric 2-square matrices:

$$
m_{A B} \longrightarrow m_{A B} m_{C D} \tilde{\lambda}^{A C} \tilde{\lambda}^{B D}=\left(m_{C D} \tilde{\lambda}^{C D}\right)^{2}+\operatorname{det}\left(m_{C D}\right) .
$$

It can be easily seen that it is non-degenerate and has signature $(++-)$. We can then complete a base $\left\{\tilde{\lambda}_{A B}, q_{A B}, w_{A B}\right\}$ in this space of symmetric matrices such that

$$
\left.\begin{array}{l}
q_{A B} \tilde{\lambda}^{A B}=0, \quad q_{A B} \tilde{\lambda}^{A C} \tilde{\lambda}^{B D} q_{C D}=-w_{A B} \tilde{\lambda}^{A C} \tilde{\lambda}^{B D} w_{C D}=2 q, \\
w_{A B} \tilde{\lambda}^{A B}=0, \quad w_{A B} \tilde{\lambda}^{A C} \tilde{\lambda}^{B D} q_{C D}=2(|q|-1)
\end{array}\right\}
$$

and, besides, $\operatorname{det}\left(q_{A B}\right)=-\operatorname{det}\left(w_{A B}\right)=q$ and $\operatorname{det}\left(\tilde{\lambda}_{A B}\right)=-1$.

$\left\{\tilde{\lambda}_{A B}, q_{A B}, w_{A B}\right\}$ is thus a rigid base for the quadratic form (B.10): an orthogonal base in the case $q \neq 0$ and a base containing two conjugate null vectors in the case $q=0$. In all instances, $w_{A B}$ is thoroughly determined by $\tilde{\lambda}_{A B}$ and $q_{A B}$. We thus have the following differential equations (the first one comes from (B.8)):

$$
\left.\begin{array}{rl}
\frac{\mathrm{d} \tilde{\lambda}_{A B}}{\mathrm{~d} f}=q_{A B}, & \frac{\mathrm{d} q_{A B}}{\mathrm{~d} f}=q \tilde{\lambda}_{A B}+q_{A B}(1-|q|) u+q w_{A B} v \\
\frac{\mathrm{d} w_{A B}}{\mathrm{~d} f} & =(|q|-1) \tilde{\lambda}_{A B}+q q_{A B} v-w_{A B}(1-|q|) u
\end{array}\right\},
$$

where $q= \pm 1$ or 0 and $u(f)$ and $v(f)$.

As the quadratic form (B.10) can be associated with a non-degenerate metric product in the 3-space of symmetric 2-square matrices, these equations can be seen as a sort of 'Frénet-Serret equations'.

In the case $q=0$, they yield

$$
\frac{\mathrm{d} \tilde{\lambda}_{A B}}{\mathrm{~d} f}=q_{A B}, \quad \frac{\mathrm{d} q_{A B}}{\mathrm{~d} f}=u q_{A B}, \quad \frac{\mathrm{d} w_{A B}}{\mathrm{~d} f}=-\tilde{\lambda}_{A B}-u w_{A B}
$$

whose solution is

$$
\tilde{\lambda}_{A B}=\hat{\lambda}_{A B}+F \hat{q}_{A B}, \quad q_{A B}=\dot{F} \hat{q}_{A B},
$$

where $\hat{\lambda}_{A B}$ and $\hat{q}_{A B}$ are constant matrices satisfying (B.11), $F=F(f)$ and $\dot{F}:=\mathrm{d} F / \mathrm{d} f$; whereas in the case $q= \pm 1$, (B.12) reads

$$
\frac{\mathrm{d} \tilde{\lambda}_{A B}}{\mathrm{~d} f}=q_{A B}, \quad \frac{\mathrm{d} q_{A B}}{\mathrm{~d} f}=q \tilde{\lambda}_{A B}+q v w_{A B}, \quad \frac{\mathrm{d} w_{A B}}{\mathrm{~d} f}=q v q_{A B}
$$

The condition $R_{2}=0$. From (B.8) and (21) it easily follows that

$$
R_{2}=\frac{1}{2} h^{b c}\left(t_{b} t_{c}-q f_{b} f_{c}\right) \quad \text { with } \quad t:=\log \tau,
$$

where (B.9) and (B.11) have been used. Now, as $h_{b c}$ is positive definite, the condition $R_{2}=0$ allows two different cases:

$$
\left.\begin{array}{l}
\text { if } q=0, \text { then } \quad t_{b}=0 \text { and } \tau=\text { constant } \\
\text { if } q=1, \text { then } \quad h^{b c} t_{b} t_{c}=h^{b c} f_{b} f_{c}
\end{array}\right\} .
$$

The case $q=-1$ is forbidden, because $R_{2}=0$ would imply $f_{b}=0$ and $\mathrm{d} \tilde{\lambda}_{A B}=0$, which amounts to $q_{A B}=0$, in contradiction with $\operatorname{det}\left(q_{A B}\right)=-1$.

The condition $P_{D c A b}=0$.

For $q=0$ on account of (B.14) and (B.17), $P_{D c A b}=0$ implies that $D_{b} F_{c}=0$. Hence, using (B.14) and (B.17), we have that

$\lambda_{A B}=\frac{\tau_{0}}{\sqrt{2}}\left[\hat{\lambda}_{A B}+F \hat{q}_{A B}\right] \quad$ with $\quad D_{a b} F=0, \quad \tau=\tau_{0}=$ constant. 
For $q=1$ substituting (B.15) into (25) and using (B.8), (B.9) and (B.11), we obtain that $P_{D c A b}=0$ amounts to

$$
\mathcal{T}_{b c}:=D_{b} t_{c}+\frac{1}{2} t_{b} t_{c}+\frac{1}{2} f_{b} f_{c}=0, \quad \mathcal{F}_{b c}:=D_{b} f_{c}+t_{(b} f_{c)}=0
$$

supplemented with $v=0$.

Using this, equations (B.15) read

whose solution is

$$
\frac{\mathrm{d} \tilde{\lambda}_{A B}}{\mathrm{~d} f}=q_{A B}, \quad \frac{\mathrm{d} q_{A B}}{\mathrm{~d} f}=\tilde{\lambda}_{A B}, \quad \frac{\mathrm{d} w_{A B}}{\mathrm{~d} f}=0
$$

$$
\tilde{\lambda}_{A B}=\hat{\lambda}_{A B} \cosh f+\hat{q}_{A B} \sinh f,
$$

where $\hat{\lambda}_{A B}$ and $\hat{q}_{A B}$ are constant matrices satisfying (B.11).

Now, equation (B.19) is a partial differential system where all the derivatives of the unknowns $t$ and $f$ are specified. The subsequent integrability conditions do not imply any new condition. Moreover, equation (B.16) implies a further restriction

$$
\frac{1}{2} h^{b c}\left(t_{b} t_{c}-f_{b} f_{c}\right)=0
$$

which is compatible with (B.19); indeed, $D_{a} R_{2}+t_{a} R_{2}=0$, and provided that $R_{2}$ vanishes at $x_{0} \in \mathcal{S}$ it vanishes in some open neighbourhood of $x_{0}$.

We now introduce the new variables $\Phi_{ \pm}:=\mathrm{e}^{(t \pm f) / 2}$ and equations (B.19) and (B.22) become

$$
D_{b c} \Phi_{ \pm}=0 \quad \text { and } \quad h^{b c} D_{b} \Phi_{+} D_{c} \Phi_{-}=0 .
$$

From (B.21) it then follows immediately that

$$
\lambda_{A B}=2^{-3 / 2}\left(\Phi_{+}^{2}\left[\hat{\lambda}_{A B}+\hat{q}_{A B}\right]+\Phi_{-}^{2}\left[\hat{\lambda}_{A B}-\hat{q}_{A B}\right]\right) \quad \tau=\Phi_{+} \Phi_{-} .
$$

With a little algebra it can be seen that, as a consequence of (B.11), there exist $\hat{m}_{A}$ and $\hat{n}_{A}$ such that $\hat{m}_{2} \hat{n}_{1}-\hat{m}_{1} \hat{n}_{2}=1$ and that

$$
\hat{\lambda}_{A B}+\hat{q}_{A B}=2 \hat{n}_{A} \hat{n}_{B} \quad \text { and } \quad \hat{\lambda}_{A B}-\hat{q}_{A B}=-2 \hat{m}_{A} \hat{m}_{B} .
$$

Therefore, (B.24) finally yields

$$
\lambda_{A B}=\frac{1}{\sqrt{2}}\left(-\Phi_{-}^{2} \hat{m}_{A} \hat{m}_{B}+\Phi_{+}^{2} \hat{n}_{A} \hat{n}_{B}\right)
$$

with $\Phi_{ \pm}$fulfilling (B.23). As a consequence, it follows from (4) that $\tau=\Phi_{+} \Phi_{-}$.

\section{B.1. Summary: How to proceed? Guidelines}

We start from a given Riemannian metric $h_{a b}$ on $\mathcal{S}$.

(1) If $h_{a b}$ is flat, then we take $\theta_{A}=0$ and

(a) choose two matrices $\hat{\lambda}_{A B}, \hat{q}_{A B}$ fulfilling (B.11), with $q:=\operatorname{det}\left(\hat{q}_{A B}\right)$;

(b) then, $\lambda_{A B}$ is given by (B.26) if $q=1$ or by (B.18) if $q=0$.

(2) If $h_{a b}$ is not flat, we first check whether $\mathcal{R}$ fulfils (B.7). If so, we choose two constants $\alpha \neq 0$ and $C \neq 0$ and take (equation (B.5))

$$
\tau=\left(-\frac{3 \alpha C^{2}}{\mathcal{R}}\right)^{1 / 4}, \quad \theta=\frac{C}{\tau}=\left(-\frac{C^{2} \mathcal{R}}{3 \alpha}\right)^{1 / 4},
$$

then choose $k_{A}$ and $\hat{\lambda}_{A B}$ such that $\hat{\lambda}^{A B} k_{A} k_{B}=\alpha$ (equation (B.4)) and take $\theta_{A}=k_{A} \theta$, $\theta^{A}=-\frac{2 C}{\tau^{3}} \hat{\lambda}^{A B} k_{B}, \quad \lambda_{A B}=k_{A} k_{B} \lambda+\hat{\lambda}_{A B} \quad$ with $\quad \lambda:=-\frac{1}{\alpha}\left(\frac{\tau^{2}}{2}+\delta_{0}\right)$.

In both cases the covectors $\xi^{A} \in \Lambda^{1} \mathcal{M}$ can be determined as indicated in subsection 2.4, i.e. by solving equations (26) and (27). 


\section{Appendix C.}

As the horizontal metrics $h_{a b}$ and $\bar{h}_{a b}$ are both elliptical, there exists an $\bar{h}$ orthonormal frame $\{\mu, \nu\}$ in $\Lambda^{1} \mathcal{S}$, with dual $\{\mathbf{v}, \mathbf{w}\}$, such that

$$
h_{a b}=\zeta_{1} \mu_{a} \mu_{b}+\zeta_{2} v_{a} v_{b} \quad \text { and }
$$

the components of the shift covectors are

$$
\kappa_{a}^{A}=m^{A} \mu_{a}+n^{A} v_{a}
$$

and equations (39)-(41) yield

$$
\zeta_{\alpha}-(2 a+b)+\frac{a(a+b)}{\zeta_{\alpha}}+x_{\alpha}=0, \quad \alpha=1,2,
$$

with $x_{1}:=\lambda_{A B} m^{A} m^{B}$ and $x_{2}:=\lambda_{A B} n^{A} n^{B}$

$\lambda_{A B} m^{A} n^{B}=0$

$\lambda_{A B} m^{B}-\frac{a(a+b)}{\zeta_{1}} \bar{\lambda}_{A B} m^{B}=0 \quad$ and $\quad \lambda_{A B} n^{B}-\frac{a(a+b)}{\zeta_{2}} \bar{\lambda}_{A B} n^{B}=0$

and

$\lambda_{A B}-(2 a+b) \bar{\lambda}_{A B}+a(a+b) \bar{\lambda}_{A C} \bar{\lambda}_{B D} \lambda^{C D}+\lambda_{B D} m^{D} \bar{\lambda}_{A C} m^{C}+\lambda_{B D} n^{D} \bar{\lambda}_{A C} n^{C}=0$.

In the generic case, $m^{1} n^{2}-m^{2} n^{1} \neq 0$, which we shall assume ${ }^{7}$, equation (C.6) is implied by (C.4) and (C.5). Therefore, it follows that

Proposition 4. The metrics $\eta_{a b}$ and $g_{a b}$, given by (36) in terms of $h_{a b}, \lambda_{A B}, \xi_{a}^{A}=\bar{\xi}_{a}^{A}+\kappa_{a}^{A}$, $\bar{h}_{a b}, \bar{\lambda}_{A B}$ and $\bar{\xi}_{a}^{A}$, are connected by the deformation law (34) if, and only if, (C.3), (C.4) and (C.5) are fulfilled. (Provided that $\kappa_{a}^{1}$ and $\kappa_{a}^{2}$ are linearly independent.)

\section{C.1. The unknowns}

Relations (C.5) can be written as

$$
\bar{\lambda}_{A B} \lambda^{B C} m_{C}=\frac{\zeta_{1}}{a(a+b)} m_{A} \quad \text { and } \quad \bar{\lambda}_{A B} \lambda^{B C} n_{C}=\frac{\zeta_{2}}{a(a+b)} n_{A}
$$

where $m_{A}:=\lambda_{A B} m^{B}$ and $n_{A}:=\lambda_{A B} n^{B}$. Therefore, $\zeta_{\alpha}=a(a+b) Z_{\alpha}$, where $Z_{\alpha}, \alpha=1,2$, are the roots of the characteristic polynomial

$$
\operatorname{det}\left(\bar{\lambda}_{A B} \lambda^{B C}-Z \delta_{A}^{C}\right):=Z^{2}-\left(\bar{\lambda}_{A B} \lambda^{A B}\right) Z+\bar{\tau}^{2} / \tau^{2}=0
$$

or $\zeta_{\alpha}=Z_{\alpha} a(a+b), \alpha=1,2$, with

$$
Z_{1,2}=\frac{1}{2}\left(\bar{\lambda}_{A B} \lambda^{A B} \pm \sqrt{\left(\bar{\lambda}_{A B} \lambda^{A B}\right)^{2}-4 \bar{\tau}^{2} / \tau^{2}}\right) .
$$

Taking this into account and using (C.8), (C.3) yields

$$
x_{\alpha}=Z_{\alpha}\left[\frac{\tau^{2}}{\bar{\tau}^{2}}-a(a+b)\right]+2 a+b-\frac{\tau^{2} \bar{\lambda}_{A B} \lambda^{A B}}{\bar{\tau}^{2}} .
$$

As, by (31), $\lambda_{A B}$ (and also $\tau$ and $\lambda_{A B}$ ) depends on the unknowns $\Phi_{ \pm}$, equations (C.9) and (C.10) are expressions of $\zeta_{\alpha}$ and $x_{\alpha}, \alpha=1,2$, in terms of $\Phi_{ \pm}, a$ and $b$.

7 We are entitled to restrict to this case whenever we can find a solution to (34). 
On its turn (C.4) implies that

$$
\lambda_{A B}=\frac{1}{x_{1}} m_{A} m_{B}+\frac{1}{x_{2}} n_{A} n_{B}
$$

with $m_{A}:=\lambda_{A B} m^{B}$ and $n_{A}:=\lambda_{A B} n^{B}$. (As the generic case has been assumed, the hyperbolicity of $\lambda_{A B}$ implies that $x_{1} x_{2}<0$, and a suitable choice of $\mu_{a}$ and $v_{b}$ permits to take $x_{1}<0$ and $x_{2}>0$, in all generality.)

Now, comparing (C.11) and (31), it results that there exists $\varphi \in \Lambda^{0} \mathcal{S}$ such that

$$
\left.\begin{array}{l}
m_{A}=\sqrt[4]{x_{1}^{2} / 2}\left(\Phi_{-} \cosh \varphi \hat{m}_{A}+\Phi_{+} \sinh \varphi \hat{n}_{A}\right) \\
n_{A}=\sqrt[4]{x_{2}^{2} / 2}\left(\Phi_{-} \sinh \varphi \hat{m}_{A}+\Phi_{+} \cosh \varphi \hat{n}_{A}\right)
\end{array}\right\} .
$$

The inverse matrix $\lambda^{A B}$ is

$$
\lambda^{A B}=\frac{\sqrt{2}}{\Phi_{+}^{2}} \stackrel{*^{A}}{m} \stackrel{* B}{m}-\frac{\sqrt{2}}{\Phi_{-}^{2}} \stackrel{*^{A}}{n} \stackrel{* B}{n},
$$

where $\stackrel{* A}{m}=\left(\hat{m}_{2},-\hat{m}_{1}\right)$ and $\stackrel{* A}{n}=\left(-\hat{n}_{2},-\hat{n}_{1}\right)$. (Note that $\stackrel{* A}{m} \hat{m}_{A}=\stackrel{*^{A}}{n} \hat{n}_{A}=0$ and $\stackrel{*}{m} \hat{n}_{A}=\stackrel{*^{A}}{n} \hat{m}_{A}=1$.).

Hence, the components of the shift covectors $\kappa_{a}^{A}$ in the frame $\left\{\mu_{a}, v_{a}\right\}$ are

$$
\left.\begin{array}{l}
m^{A}=\sqrt[4]{2 x_{1}^{2}}\left(-\frac{\cosh \varphi}{\Phi_{-}} \stackrel{* A}{n}^{A}+\frac{\sinh \varphi}{\Phi_{+}} \stackrel{*}{m}\right) \\
n^{A}=\sqrt[4]{2 x_{2}^{2}}\left(-\frac{\sinh \varphi}{\Phi_{-}} \stackrel{*}{n}^{A}+\frac{\cosh \varphi}{\Phi_{+}} \stackrel{*}{m}^{A}\right)
\end{array}\right\},
$$

which, taking (C.9) and (C.10) into account, give $m^{A}$ and $n^{A}$ in terms of the unknowns $\Phi_{ \pm}, a$, $b$ and $\varphi$. Note that

$$
m^{1} n^{2}-m^{2} n^{1}=\sqrt{-x_{1} x_{2}} /\left(\Phi_{+} \Phi_{-}\right)
$$

Furthermore, combining (C.5) and (C.14), we obtain after some algebra that

$$
\sinh 2 \varphi=\frac{2^{3 / 2}}{\left(Z_{1}-Z_{2}\right) \Phi_{+} \Phi_{-}} \bar{\lambda}_{A B} \stackrel{*}{m} \stackrel{A_{*} B}{n},
$$

which determines $\varphi$ in terms of $\Phi_{ \pm}, a, b$ and the datum $\bar{\lambda}_{A B}$.

Let us turn now our attention to the unknown frame $\{\mu, v\}$. Since it is $\bar{h}$-orthonormal, if we choose an arbitrary $\bar{h}$-orthonormal frame $\left\{\alpha^{1}, \alpha^{2}\right\}$, with dual $\left\{\mathbf{e}_{1}, \mathbf{e}_{2}\right\}$, there exists $\psi \in \Lambda^{0} \mathcal{S}$ such that

$$
\mu=\cos \psi \alpha^{1}-\sin \psi \alpha^{2}, \quad v=\sin \psi \alpha^{1}+\cos \psi \alpha^{2}
$$

and

$$
\mathbf{v}=\cos \psi \mathbf{e}_{1}-\sin \psi \mathbf{e}_{2}, \quad \mathbf{w}=\sin \psi \mathbf{e}_{1}+\cos \psi \mathbf{e}_{2}
$$

We have proved so far that, if $\eta_{a b}$ and $g_{a b}$ are given by (36) and relations (C.3) to (C.6) are fulfilled, then five functions, $\Phi_{ \pm}, a, b$ and $\psi$ in $\Lambda^{0} \mathcal{S}$, exist such that

(A) $h_{a b}$ and $\kappa_{a}^{A}$ are given by (C.1) and (C.2), with $m^{A}, n^{A}, \mu_{a}$ and $v_{a}$ given by (C.14) and (C.17) in terms of these five functions and a chosen $\bar{h}$-orthonormal frame $\left\{\alpha^{1}, \alpha^{2}\right\}$ and (B) $\lambda_{A B}$ is given by (31). 
The converse also holds, that is, if (A) and (B) are fulfilled, then $\eta_{a b}$ and $g_{a b}$ defined by (36) fulfil conditions (C.3) to (C.6), and are therefore connected by the deformation law (34), for any $\Phi_{ \pm}, a, b$ and $\psi$.

We shall now write equations (32), (42) and $\mathcal{R}=0$ in terms of these new unknowns. As for the first one, it is already written in terms of $\Phi_{ \pm}$, that is,

$$
\mathcal{F}_{a b}^{ \pm}:=D_{a b} \Phi_{ \pm}=0, \quad R_{2}:=h^{a b} D_{a} \Phi_{+} D_{b} \Phi_{-}=0 .
$$

\section{C.2. Equation (42)}

As the frame $\{\mu, v\}$ is $\bar{h}$-orthonormal, we have that

$$
\bar{D}_{a} \mu_{b}=\Omega_{a} v_{b} \quad \text { and } \quad \bar{D}_{a} v_{b}=-\Omega_{a} v_{b},
$$

where $\bar{D}$ is the Levi-Civita connection for $\bar{h}_{a b}$ and $\Omega \in \Lambda^{1} \mathcal{S}$ is the rotation of the $\bar{h}$-orthonormal frame. We have therefore that

$$
\mathrm{d} \mu=\Omega \wedge \nu \quad \text { and } \quad \mathrm{d} \nu=-\omega \wedge \mu .
$$

Using now (C.17), equations (C.20) yield

$$
\mathcal{E}:=\mathrm{d} \psi+\Omega+v_{d} \mu_{c} \bar{\gamma}_{a b}^{c} \bar{h}^{c d} \alpha^{a}=0
$$

where $\bar{\gamma}_{a b}^{c}$ are the $\bar{D}$-connection coefficients in the given frame $\left\{\alpha^{1}, \alpha^{2}\right\}$.

Now, writing $\Omega=\Omega_{1} \mu+\Omega_{2} v$ and using (C.2) and (C.21), equations (42) lead to

$$
\Omega_{1} m^{A}+\Omega_{2} n^{A}=\rho^{A}, \quad \text { with } \quad \rho^{A}:=-\bar{\theta}^{A}+\left(\mathbf{w} m^{A}\right)-\left(\mathbf{v} n^{A}\right),
$$

where $\{\mathbf{w}, \mathbf{v}\}$ is the dual frame for $\{\mu, \nu\}$, and it can be solved to obtain

$\Omega_{1}=\frac{\Phi_{+} \Phi_{-}}{\sqrt{-x_{1} x_{2}}}\left(\rho^{1} n^{2}-\rho^{2} n^{1}\right), \quad \Omega_{2}=-\frac{\Phi_{+} \Phi_{-}}{\sqrt{-x_{1} x_{2}}}\left(\rho^{2} m^{1}-\rho^{1} m^{2}\right)$,

where (C.15) has been included.

Once $\Omega$ is known, it can be substituted into (C.22) which becomes an equation which determines $\mathrm{d} \psi$. The subsequent integrability condition then reads

$$
\mathrm{d} \Omega+\frac{1}{2} \overline{\mathcal{R}} \bar{\epsilon}=0
$$

which is integrable because $\operatorname{dim} \mathcal{S}=2$ and amounts to

$$
\mathcal{W}:=\left(\mathbf{v} \Omega_{2}\right)-\left(\mathbf{w} \Omega_{1}\right)+\Omega_{b} \Omega_{c} \bar{h}^{b c}+\frac{1}{2} \overline{\mathcal{R}}=0 .
$$

\section{C.3. The flatness of $h_{a b}$}

Consider the frame $\left\{\theta^{1}=\mu, \theta^{2}=v\right\}$ and its dual frame $\left\{\mathbf{v}_{1}=\mathbf{v}, \mathbf{v}_{2}=\mathbf{w}\right\}$. If we define $\gamma_{a b}:=h^{a c} \gamma_{b}^{c}$, where $\gamma_{b}^{c}$ are the connection forms for $D$, we have

$$
\begin{aligned}
& \gamma_{a a}=\frac{1}{2} \mathrm{~d} \zeta_{a} \quad \text { and } \\
& \gamma_{12}=-\gamma_{21}=\left(-\zeta_{1} \Omega_{1}+\frac{1}{2}\left(\mathbf{w} \zeta_{1}\right)\right) \mu-\left(\zeta_{2} \Omega_{2}+\frac{1}{2}\left(\mathbf{v} \zeta_{2}\right)\right) \mu,
\end{aligned}
$$

where (C.21), (C.1) and the fact that $\gamma_{12}+\gamma_{21}=0$ have been included.

As $\operatorname{dim} \mathcal{S}=2$, only one of the curvature forms, $\Theta_{b}^{a}:=\mathrm{d} \gamma_{b}^{a}+\gamma_{c}^{a} \wedge \gamma_{b}^{c}$, is independent. The flatness of $h_{a b}$ therefore amounts to $h_{1 c} \Theta_{2}^{c}-h_{2 c} \Theta_{1}^{c}=0$ which, after some algebra, yields

$$
\mathrm{d}\left(\frac{1}{\sqrt{\zeta_{1} \zeta_{2}}} \gamma_{12}\right)=0
$$

which can be expanded as

$$
\begin{aligned}
\mathcal{T}:=\zeta_{1}\left(\mathbf{w} \Omega_{1}\right) & -\zeta_{2}\left(\mathbf{v} \Omega_{2}\right)-\frac{1}{2}\left[\left(\mathbf{w}^{2} \zeta_{1}\right)+\left(\mathbf{v}^{2} \zeta_{2}\right)\right] \\
& -\zeta_{1} \Omega_{1}^{2}-\zeta_{2} \Omega_{2}^{2}+\frac{3}{2} \Omega_{1}\left(\mathbf{w} \zeta_{1}\right)-\frac{3}{2} \Omega_{2}\left(\mathbf{v} \zeta_{2}\right) .
\end{aligned}
$$




\section{References}

[1] Llosa J and Soler D 2005 Class. Quantum Grav. 22893

[2] Llosa J and Carot J 2009 Class. Quantum Grav. 26055013

[3] Garcia-Parrado A and Senovilla J M M 2004 Class. Quantum Grav. 212153

[4] Kobayashi S and Nomizu K 1969 Foundations of Differential Geometry (New York: Interscience)

[5] Ferrando J J and Saez J A 2004 J. Math. Phys. 45652

[6] Rainich G Y 1925 Trans. Am. Math. Soc. 27106

[7] Geroch R 1971 J. Math. Phys. 12918

[8] Geroch R 1972 J. Math. Phys. 13394

[9] Stephani H 2004 Relativity (Cambridge: Cambridge University Press) equation (33.22)

[10] Choquet-Bruhat Y, DeWitt-Morette C and Dillard-Bleick M 1987 Analysis, Manifolds and Physics (Amsterdam: North-Holland)

[11] Eisenhart L P 1997 Riemannian Geometry (Princeton, NJ: Princeton University Press) equation (28.7)

[12] Stefani H, Kramer D, MacCallum M, Hoenselaers C and Herlt E 2003 Exact Solutions of Einstein's Field Equations (Cambridge: Cambridge University Press) equation (19.21)

[13] John F 1982 Partial Differential Equations (Berlin: Springer) 